\title{
Transient Analysis of
} Electromagnetic Reflection From Dispersive Materials 
he National Bureau of Standards ${ }^{1}$ was established by an act of Congress on March 3, 1901. The Bureau's overall goal is to strengthen and advance the nation's science and technology and facilitate their effective application for public benefit. To this end, the Bureau conducts research and provides: (1) a basis for the nation's physical measurement system, (2) scientific and technological services for industry and government, (3) a technical basis for equity in trade, and (4) technical services to promote public safety. The Bureau's technical work is performed by the National Measurement Laboratory, the National Engineering Laboratory, the Institute for Computer Sciences and Technology, and the Center for Materials Science.

\section{The National Measurement Laboratory}

Provides the national system of physical and chemical measurement; coordinates the system with measurement systems of other nations and furnishes essential services leading to accurate and uniform physical and chemical measurement throughout the Nation's scientific community, industry, and commerce; provides advisory and research services to other Government agencies; conducts physical and chemical research; develops, produces, and distributes Standard Reference Materials; and provides calibration services. The Laboratory consists of the following centers:
- Basic Standards ${ }^{2}$

- Radiation Research

- Chemical Physics

- Analytical Chemistry

\section{The National Engineering Laboratory}

Provides technology and technical services to the public and private sectors to address national needs and to solve national problems; conducts research in engineering and applied science in support of these efforts; builds and maintains competence in the necessary disciplines required to carry out this research and technical service; develops engineering data and measurement capabilities; provides engineering measurement traceability services; develops test methods and proposes engineering standards and code changes; develops and proposes new engineering practices; and develops and improves mechanisms to transfer results of its research to the ultimate user. The Laboratory consists of the following centers:
- Applied Mathematics

- Electronics and Electrical Engineering ${ }^{2}$

- Manufacturing Engineering

- Building Technology

- Fire Research

- Chemical Engineering ${ }^{2}$

\section{The Institute for Computer Sciences and Technology}

Conducts research and provides scientific and technical services to aid Federal agencies in the selection, acquisition, application, and use of computer technology to improve effectiveness and economy in Government operations in accordance with Public Law 89-306 (40 U.S.C. 759), relevant Executive Orders, and other directives; carries out this mission by managing the Federal Information Processing Standards Program, developing Federal ADP standards guidelines, and managing Federal participation in ADP voluntary standardization activities; provides scientific and technological advisory services and assistance to Federal agencies; and provides the technical foundation for computer-related policies of the Federal Government. The Institute consists of the following centers:
- Programming Science and Technology

- Computer Systems Engineering

\section{The Center for Materials Science}
Conducts research and provides measurements, data, standards, reference materials, quantitative understanding and other technical information funda- mental to the processing, structure, properties and performance of materials; addresses the scientific basis for new advanced materials technologies; plans research around cross-country scientific themes such as nondestructive
- Inorganic Materials
- Fracture and Deformation ${ }^{3}$
- Polymers
- Metallurgy
- Reactor Radiation evaluation and phase diagram development; oversees Bureau-wide technical programs in nuclear reactor radiation research and nondestructive evaluaion; and broadly disseminates generic technical information resulting from its programs. The Center consists of the following Divisions:

\footnotetext{
'Headquarters and Laboratories at Gaithersburg, MD, unless otherwise noted; mailing address Caithersburg, MD 20899

Some divisions within the center are located at Boulder, CO 80303.

L.ocated at Boulder, $\mathrm{CO}$, with some elements at Gaithersburg, MD.
} 


\section{Transient Analysis of Electromagnetic Reflection From Dispersive Materials}

\section{A. George Lieberman}

Center for Manufacturing Engineering

National Engineering Laboratory

National Bureau of Standards

Gaithersburg, MD 20899

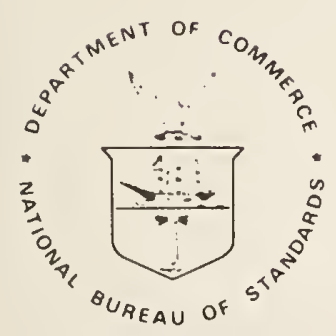

U.S. DEPARTMENT OF COMMERCE, Malcolm Baldrige, Secretary NATIONAL BUREAU OF STANDARDS, Ernest Ambler, Director 
National Bureau of Standards Technical Note 1202

Natl. Bur. Stand. (U.S.), Tech. Note 1202, 70 pages (March 1985) CODEN: NBTNAE

U.S. GOVERNMENT PRINTING OFFICE

WASHINGTON: 1985

For sale by the Superintendent of Documents. U.S. Government Printing Office. Washington. DC 20402 


\section{TRANSIENT ANALYSIS DF ELECTROMAGNETIC}

\section{REFLECTION FROM DISPERSIVE MATERIALS}

A. George Lieberman

National Bureau of Standards

Washington, DC 20234

\section{ABSTRACT}

Theoretical expressions are presented describing the transient and steady-state temporal evolution of an impulsive, TE-polarized, plane wave reflected into vacuum from any of a variety of frequency-dispersive material surfaces. Polar dielectrics, non-polar dielectrics, metals and plasmas are treated using, respectively, the Debye, Lorentz, and Drude material models to investigate the effects of dispersion upon dimensional measurements performed with optical pulses of extremely short duration. The more general problem, concerning the reflection of an optical pulse of any specified waveform, is resolved by performing a convolution of the incident wave at the material surface with the previously determined reflection of an impul sive wave.

Key words: Debye dielectric; dispersion, Drude metal; electromagnetic fields; Green"s function; impulse response; Lorentz dielectric; material models; optical pulses; plasma; reflected waves; transient fields; waveform analysis. 


\section{CONTENTS}

Page

1. INTRODUCTION 1

2. DIELECTRIC THEORY 3

2. 1 Terminology 3

2.2 The Lorentz Dielectric $\quad 7$

2.3 The Debye Dielectric 16

3. PLANE WAVE PROPAGATION AND REFLECTION 22

4. INTEGRAL TRANSFORMATIONS AND THE FRESNEL THEORY 30

5. CONSTRUITION OF THE TRANSIENT PROPAGATOR 37

6. THE TRANSIENT PROPAGATOR FOR TREATING REFLECTIONS FROM A LORENTZ DIELECTRIC $\quad 40$

7. THE TRANSIENT PROPAGATOR FOR TREATING REFLECTIONS FROM A DRUDE METAL AND A PLASMA

8. THE TRANSIENT PROPAGATOR FOR TREATING REFLECTIONS FROM A DEEYE DIELECTRIC

55

9. SUMMARY AND CONCLUSIONS 60

10. ACKNOWLEDGEMENT 63

11. REFERENCES 64 


\section{INTRODUCTION}

A great deal of effort has been expended in understanding the harmonic time-dependent solutions of Maxwell's equations. This has happened for a number of good reasons, but primarily because Fourier's integral theorem permits a source of fairly arbitary time dependence to be pictured as a superposition of harmonic oscillators. If the field intensities remain moderate the radiation from these oscillators can be treated independently, and if the spectral width of the original source is sufficiently 1 imited and removed from the resonances of the supporting medium then all harmonics will propagate with the same phase velocity. Under these conditions the medium is said to behave non-dispersively and the time dependence of the radiated waveform at a distant location will reproduce that of the source.

With the recent advent of 1 aser sources capable of generating femtosecond pulses $[1]^{1}$ (containing only a few cycles of oscillation), and with the expectation of generating still shorter pulses in the future, the dispersive properties of a medium can now hardly be ignored. With this in mind, a study of the transient and steady-state aspects of electromagnetic pulse reflection from dispersive materials was undertaken.

A review of the archival literature disclosed a considerable volume of research treating the propaggatigㅡㅁ of optical pulses in dispersive media. This research commences with the theoretical demonstration by Sommerfeld and Brillouin [2] that the velocity of a signal cannot exceed the velocity of light in a region of

1 Numbers in brackets indicate 1 iterature references 1 isted at the end of this report. 
anomalous dispersion. In contrast, the problem of optical pulse reeㅛ르드늠 from dispersive media has hardly been explored. The little that has been published deals, on one hand, with dissipative materials for which the constitutive parameters do not depend upon frequency [3,4]. Dispersion in that unrealistic case is caused by relative changes between the frequencydependent displacement current and the frequency-independent conduction current. On the other hand, the reflection of radio frequency pulses from the ionosphere [5-9] has received much more attention but its applicability to optics is diminished by the frequency range and material models which have been studied. The results reported in this paper include the above as special cases. The material models devised by Debye [10], Lorentz [11], and Drude [12], on the basis of classical electron theory, are employed here to study optical pulse relections from dielectric, metal and plasma surfaces. 


\section{DIELECTRIC THEORY}

\section{1 Terminology}

The behavior of electromagnetic radiation is governed by the Maxwell equations, the boundary conditions on the field vectors, and the constitutive relations describing the supporting medium. When, as in the present case, the interatomic material forces are considered short-ranged or weak the constitutive relations assume a local spatial character and also become independent of the boundary conditions. Such a medium may, nevertheless, exhibit a global temporal character in the sense of retaining a memory of its earlier history, including initial conditions. This situation arises whenever the relaxation times characterizing the medium are comparable to the period of the radiation, a condition satisfied, for example, in an optical field by the motion of valence electrons in atoms.

For a linear, isotropic dielectric with memory the present value of the polarization field $\vec{P}(t)$ is determined by an integral over all previous values of the impressed electric field $\vec{E}(t)$ :

$$
\vec{P}(t)=\int_{-\infty}^{t} \epsilon_{0} x(t-\tau) \vec{E}(\tau) d \tau .
$$

Both $\vec{P}(t)$ and $\vec{E}(t)$ are, of course, to be evaluated at the same spatial point due to the local character of the medium.

The kernel, $\epsilon_{0} \chi(t)$, of integral equation (2-1) has special significance in that it describes the response of the dielectric to an elementary impulsive electric field. Certainly, if the magnitude of the electric field is given by $E(t)=\delta(t)$, where 
$\delta(t)$ is the Dirac delta function, then the polarization field is given by $P(t)=\varepsilon_{0} x(t)$. Function $\chi(t)$ also has the general property that it is finite for all values of time, including $t=0$, and tends to zero as $t^{-t_{\infty}}$. The latter simply expresses the fact that the polarization cannot be appreciably affected by values of the electric field at sufficiently remote times. In fact, $x(t)$ differs from zero only for periods of the order of the relaxation times characterizing the underlying molecular polarization processes. All materials possess some degree of memory; the hysteresis exhibited by magnetically polarized iron is a dramatic example.

Equation (2-1) has the form of a convolution integral in the time domain. However, under a Fourier transformation, defined by the pair of integrals:

$$
\begin{aligned}
& \vec{F}(t)=\frac{1}{2 \pi} \int_{-\infty}^{\infty} \vec{P}(\omega) e^{+j \omega t} d \omega \\
& \vec{P}(\omega)=\int_{-\infty}^{\infty} \vec{P}(t) e^{-j \omega t} d t,
\end{aligned}
$$

eq. (2-1) can be mapped into an algebraic relation with frequency w as the independent variable. Namely,

$$
\vec{P}(\omega)=\epsilon_{0} X(\omega) \vec{E}(\omega),
$$

where $\vec{E}(\omega)$ and $\chi(\omega)$ correspond, respectively, to the fourier 
transforms of $\vec{E}(t)$ and $x(t) .^{2}$ The function $x(\omega)$, relating the dielectric polarization to the electric intensity at each frequency, is called the dielectric susceptibility while its dependence on frequency is referred to as dispersion. A material possessing a strong memory is also highly dispersive.

Conversely, a non-dispersive material (for which $X(\omega)=\chi_{0}$ is a constant) possesses no memory at all; that is to say, $x(t)=x_{0} \delta(t)$.

In either representation (time or frequency) the electric displacement field $\vec{D}$ is given by

$$
\vec{D}=\epsilon \vec{E}+\vec{P}
$$

As usual, $\epsilon_{0}$ is the dielectric constant of vacuum and $\vec{P}$ describes the effect of the material occupying that physical space. The advantage of the frequency representation becomes evident upon substituting eq. $(2-3)$ into eq. $(2-4)$ :

$$
\vec{D}(\omega)=\epsilon_{0}[1+\chi(\omega)] \vec{E}(\omega)=\epsilon(\omega) \vec{E}(\omega) .
$$

The factor $\in(\omega)$ relating $\vec{D}(\omega)$ with $\vec{E}(\omega)$ is referred to as the dielectric permittivity of the medium. It is important to again emphasize that, unless the dielectric susceptibility is non-

\section{2}

Although the same symbol is used to denote the function and its transform this should not be misconstrued to mean that the two functions are ideqtical except for their,arguments. Thus $P(\omega)$ is not the function $\vec{P}(t)$ evaluated at $t=\omega ; \vec{P}(t)$ is the fourier transform of $P(\omega)$. 
dispersive, the multiplicative structure of constitutive relation (2-5) is valid only in the frequency representation.

An expression completeiy analogous to eq. (2-5) describes the magnetization state of the given medium. Namely,

$$
\vec{B}(\omega)=\mu(\omega) \vec{H}(\omega) .
$$

However, for the vast majority of optical phenomena the dispersion attributed to magnetization can be ignored because magnetic resonances occur far below the optical frequency band. As a result, the constitutive relation in the optical frequency range joining the magnetic flux density $\vec{B}(\omega)$ to the magnetic field intensity $\vec{H}(\omega)$ is usually written

$$
\vec{B}(\omega)=\mu_{0} \vec{H}(\omega)
$$

where $\mu_{0}$ is a constant, often the permeability of vacuum.

The total current flowing through the medium consists of two components: a conduction current $\vec{J}(\omega)$ and a displacement current $j \omega \vec{D}(\omega)$. The former is described by ahm 51 aw:

$$
\vec{J}(\omega)=\sigma(\omega) \vec{E}(\omega)
$$

The 1 atter, according to eq. (2-5), is also proportional to $\vec{E}(\omega)$ and a convenient practice has been to combine the two components into a single current by introducing an effective permittivity function $\epsilon_{\text {eff }}(\omega)$. 


$$
\vec{J}(\omega)+j \omega \vec{D}(\omega)=(\sigma+j \omega \epsilon) \vec{E}(\omega)=j \omega \epsilon \text { eff }(\omega) \vec{E}(\omega)=
$$

The dispersion of a dielectric medium in the optical frequency range is therefore fully characterized by its effective permittivity function. The structure of this function depends upon the physical model describing the polarization process. The fundamental processes in dielectric polarization are: (1) distortion of atomic or molecular charge distributions by an electric field, and (2) alignment of dipolar molecules along the local electric field direction. These processes are described, respectively, by the Lorentz dielectric model and the Debye dielectric model.

\subsection{The Lorentz Dielectric Model}

Lorentz pictured dielectric polarization as resulting from the separation of positive and negative charges in the atomic structure of a substance by the application of an external electric field [11]. For excitations at optical frequencies, this polarization is ascribed to the dipole moment induced by the displacement of the valence electrons' center of charge relative to that of its atomic core. For small displacements the electrostatic restoring force within an atom is 1 inearly proportional to the relative displacement, with the result that the described motion is harmonic. Since the mass of an atomic core is at least three orders of magnitude 1 arger than that of an electron, the relative displacement $\vec{r}(t)$ can be attributed entirely to the electronic motion which is governed by the equation: 


$$
m \frac{d^{2} \vec{r}}{d t^{2}}+v m \frac{d \vec{r}}{d t}+k \vec{r}=-e \vec{E} \cdot(t) .
$$

The first term represents the inertial reaction of the electron to its accelerationg the next term describes the viscous damping of its motion resulting from dissipative interactions (inelastic collisions) with other particles; the last term on the left deals with the electrostatic restoring force. Together these three terms balance the electric force which causes the electron's di spl acement.

For $k=0$ this equation describes the motion of a free electron in an electric field, e.g. a conduction electron in a lossy metal or plasma. Note that if the electric field is suddenly removed at $t i m e t=0$ the equation of motion with $k=0$ reduces to

$$
\frac{d}{d t}\left(\frac{d \vec{r}}{d t}\right)+\nu\left(\frac{d \vec{r}}{d t}\right)=0
$$

As a consequence, the velocity of the conduction electron is viscously damped to a standstill with the damping rate determined by the relaxation time $r=1 / v$ of the medium:

$$
\frac{d \vec{r}}{d t}=\left(\frac{d \vec{r}}{d t}\right)_{t=0} e^{-t / \tau}
$$

On the other hand, if the applied field is varied sinusoidally with frequency $\omega$, and expressed as the real part of 
a complex field, viz.,

$$
\vec{E}^{\prime}(t)=\operatorname{Re} \vec{E}^{\prime}(\omega) e^{+j \omega t}
$$

then the forced motion is oscillatory, having an amplitude given by

$$
\vec{r}(t)=\operatorname{Re} \vec{r}(\omega) e^{+j \omega t}
$$

Substitution of these expressions into equation of motion (2-10) now yields:

$$
\vec{r}(\omega)=\frac{-(e / m) \vec{E}^{\prime}(\omega)}{(j \omega)^{2}+(j \omega) \nu+\omega_{0}^{2}}
$$

where $\omega_{0}=\sqrt{k / m}$ is the natural frequency of the oscillator. This displacement of the electron from its equilibrium position, in turn, produces an induced dipole having electric moment

$$
\vec{p}(\omega)=-\vec{r}(\omega)
$$

If $N$ such oscillators are present in a unit volume of this dielectric, then the polarization field is given by

$$
\vec{P}(\omega)=-\operatorname{Ner}(\omega)=\epsilon_{0} \frac{\omega_{p}^{2}}{\left(\omega_{0}^{2}-\omega^{2}\right)+j \omega \nu} \vec{E}^{\prime}(\omega),
$$

where $\omega_{p}=\sqrt{\mathrm{Ne}^{2} / \mathrm{m \epsilon}}$ is the electron plasma frequency. The physical significance of $\omega_{p}$ will become apparent when the dispersion of a metal or plasma is examined. 
More generally, a dielectric could consist of several different kinds of polarizable ascillators each classified by a particular elastic constant, effective mass, charge, relaxation time, or density. The total polarization field, in this case, would be determined as a superposition of the various component polarizations:

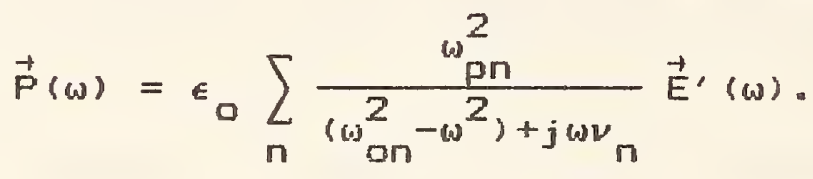

The summation is carried out over all polarization groups; each denoted by a different value of the subscript $n$. This expression can be written also in the form

$$
\vec{F}(\omega)=\epsilon_{0} \sum_{n} N_{n} \alpha_{n}(\omega) \vec{E}(\omega)
$$

or, for a single group consisting of $N$ identical atoms (oscillators) per unit volume, as

$$
\vec{P}(\omega)=\epsilon_{0} N \alpha(\omega) \vec{E}^{\prime}(\omega),
$$

where $\alpha(\omega)$ is the polarizability of a single atom.

It canno: be assumed that the local field $\vec{E}^{\prime}(\omega)$ acting on an atom is the same as the macroscopic field $\vec{E}(\omega)$ applied to the medium. There is also that electric force exerted by each atom upon every other atom of the dielectric. In the absence of an applied electric field these forces combine to create a charge distribution for which the valume polarization is usually zero. 
The application of an external field causes each atom to acquire a new polarization and to exert additional forces on every other atom. The polarization field within a given volume element now depends not only upon the direct action of the applied field but also upon its indirect action through the polarization it creates in other valume elements. For an isotropic material H. A. Lorentz [14] and L. Lorenz [15] have shown that the polarization contributes an amount $\vec{P} / 3 \in$, to the electric field, i.e.,

$$
\vec{E}^{\prime}(\omega)=\vec{E}(\omega)+\vec{P}(\omega) / 3 \epsilon \sigma^{*}
$$

This correction is also valid at a lattice point within a cubic crystal, but it is not valid for crystals of lower symmetry. For greater generality relation $(2-21)$ is expressed in the form

$$
\vec{E}^{\prime}(\omega)=\vec{E}(\omega)+k \vec{F}(\omega) / \epsilon \sigma^{g}
$$

where the proportionality constant $K$ is to be appropriately determined for a given medium.

Substituting eq. (2-22) for the local field into eq. (2-19) and solving for the polarization gives

$$
\vec{F}(\omega)=\frac{\epsilon \sum_{n} N_{n} \alpha_{n}(\omega)}{1-K \sum_{n} N_{n} \alpha_{n}(\omega)} \vec{E}(\omega) .
$$

However, according to eq. (2-3), 


$$
\vec{P}(\omega)=\epsilon_{0} X(\omega) \vec{E}(\omega)
$$

so that the dielectric susceptibility

$$
x(\omega)=\frac{\sum_{n} N_{n}{ }_{n}(\omega)}{1-K \sum_{n} N_{n} \alpha_{n}(\omega)}
$$

On comparing eqs. $(2-18),(2-19)$, and $(2-20)$ it appears that

$$
\alpha_{n}(\omega)=\frac{\omega_{\mathrm{pn}}^{2} / N_{n}}{\left(\omega_{\mathrm{on}}^{2}-\omega^{2}\right)+j \omega \nu_{n}} .
$$

Within a frequency range determined by the applied field, only a finite number of resonance terms are needed from eq. (2-24) to describe the polarization state. This being the case, $x(\omega)$ is a rational function of $\omega^{2}$ allowing eq. $(2-24)$ to be expressed in the form:

$$
\chi(\omega)=\sum_{n} \frac{\omega_{p n}^{\prime}{ }^{2}}{\left(\omega_{o n}^{\prime}{ }^{2}-\omega^{2}\right)+j \omega \nu_{n}^{\prime}}
$$

For example, when a single resonance term suffices, eq. (2-24) with the aid of eq. $(2-25)$ reduces to 


$$
x(\omega)=\frac{\omega_{p}^{2}}{\left(\omega_{0}{ }^{2}-K \omega_{p}{ }^{2}\right)-\omega^{2}+j \omega \nu} .
$$

A comparison of this expression with eq. (2-26) shows that

$$
\begin{aligned}
\omega_{p}^{\prime 2} & =\omega_{p}^{2} \\
\omega_{0}^{\prime} & =\omega_{0}^{2}-K \omega_{p}^{2} \\
\nu^{\prime} & =\nu
\end{aligned}
$$

The effect of the Lorentz-Lorenz correction to the electric field has been to shift the parameters of the Lorentz dielectric model. Since the value of $K$ is not always known, the parameters of eq. (2-26), not those of eq. $(2-18)$, are determined by measurement. For notational brevity the primes appearing on $\omega_{p}^{\prime}$ " $\omega_{a}^{\prime}$, and $\nu$ ' will hereafter be suppressed. Thus in place of (2-26) one has

$$
x(\omega)=\sum_{n} \frac{\omega_{p n}^{2}}{\left(\omega_{0}^{2}-\omega^{2}\right)+j \omega \nu_{n}} .
$$

It should be pointed out that when eq. $(2-29)$ is expressed in terms of the wavelength $\lambda=2 \pi c / \omega$, and absorption is neglected $\left(\nu_{n}=0\right)$, that this equation takes the form 


$$
n^{2}-1=\sum_{n} \frac{c_{n} \lambda^{2}}{\lambda^{2}-\lambda_{\text {on }}^{2}}=a+\sum_{n} \frac{b_{n}}{\lambda^{2}-\lambda_{o n}^{2}}
$$

where the optical refractive index is given by

$$
n^{2}=\epsilon / \epsilon_{0}=1+x
$$

according to eq. (2-5). For historical reasons eq. $(2-30)$ is referred to as Sellmeier's formula; it is widely used to empirically fit experimental dispersion data.

In view of eq. $(2-29)$ and definition (2-5) the permittivity function for the Lorentz dielectric model is given by

$$
\epsilon(\omega)=\epsilon_{0}\left[1+\sum_{n} \frac{\omega_{p n}^{2}}{\left(\omega_{o n}^{2}-\omega^{2}\right)+j \omega \nu_{n}}\right] .
$$

If the restoring force is eliminated, eq5. (2-29) and (2-32) describe the electrical behavior of a lossy metal:

$$
x(\omega)=-\sum_{n} \frac{\omega_{p n}^{2}}{\omega\left(\omega-j \nu_{n}\right)}
$$




$$
\epsilon(\omega)=\epsilon_{0}\left[1-\sum_{n} \frac{\omega_{p n}^{2}}{\omega\left(\omega-j \nu_{n}\right)}\right] .
$$

Furthermore, with $v$ set to zero, these constitutive parameters also pertain to the electrical properties of a cold, collisionless plasma. Expressions similar to eqs. (2-32) and (2-33) were originally derived by Drude [12] for a metallic conductor.

A more rigorous quantum mechanical treatment of the atomic system, given by Kramers and Hei senberg [1.3], leads to an expression for $\epsilon(6)$ which is identical in form to eq. (2-32). In the quantum mechanical case, $f_{w}$ on corresponds to the energy difference between two electronic quantum states; the reciprocal of $\nu$ relates to the 1 ifetime of a transition between these two states; and $\omega_{p n}^{2}$ is proportional to the oscillator strength for this transition. Absorption occurs through the excitation of a resonant transition and the subsequent dissipation of the excitation energy to the remainder of the system through lifetime 1 imiting mechanisms. At frequencies far from resonance, the electronic excitations (oscillators) exchange energy with the radiation field with practically no absorption taking place. This exchange does, however, lead to a modification of the propagation velocity as manifested by the refractive index $n(w)=\sqrt{\epsilon^{\prime}(w) / \epsilon}$, where $\epsilon^{\prime}$ is the real part of $\epsilon$. 


\subsection{The Debye Dielectric Model}

The literature concerning dielectric polarization is dominated by two classical theories: the Lorentz theory for resonant dispersion, and the Debye theory [10] for relaxative dispersion. The latter deals with dielectric materials containing structural elements (atoms, molecules, ionic radicals, lattice defects, etc., collectively referred to as molecules) which behave as non-resonant rotators possessing permanent electric dipole moments. These dipolar moments tend to align with the direction of an externally produced field, in opposition to the randomizing effects of thermally induced collisions. The frequency of collision is assumed to be so much larger than the rotational frequency that the molecules can be regarded as remaining stationary between collisions. As a result, the resistance to motion is dominated by viscous, rather than inertial, forces.

The original Debye calculation for the relaxation of polar dielectric liquids was based upon a consideration of weak collisions and Brownian motion. A particularly simple derivation of the Debye formula will be given here for the case of strong collisions. In a strong collision the molecule has no recollection about its orientation or state prior to the collision. The collision is also assumed to be adiabatic, i.e., to occur in a time frame much shorter than the oscillation period of the impressed field. If the collisions are both strong and adiabatic, the molecular orientations will obey a Boltzmann 
probability distribution functionally dependent upon the orientational energy of a molecular dipole within the impressed field.

Consider a dipolar molecule belonging to the class of molecules whose last collision occurred at time $t_{0}$ when the oscillating local field had magnitude $\vec{E}^{\prime}\left(t_{0}\right)=\vec{E}^{\prime} \cos w t_{0}$. If the angle between the dipolar moment $\vec{p}$ and the field $\vec{E}^{\prime}\left(t_{0}\right)$ is $\theta$, then the potential energy of this molecule is given by:

$$
\overrightarrow{-p} \cdot \vec{E}^{\prime}\left(t_{0}\right)=-p E^{\prime} \cos \omega t_{0} \cos \theta
$$

The mean polarization per molecule, obtained as an average over all dipolar orientations at the instant $t_{0}$, is directed along $\vec{E}^{\prime}$ and its magnitude is determined by the normalized Boltzmann distribution:

$$
\begin{aligned}
\langle p\rangle & =\frac{\int_{0}^{\pi} p \cos \theta \exp (x \cos \theta) \sin \theta d \theta}{\int_{0}^{\pi} \exp (x \cos \theta) \sin \theta d \theta} \\
& =\frac{p \int_{-1}^{+1} \gamma \exp (x \gamma) d \gamma}{\int_{-1}^{+1} \exp (x \gamma) d \gamma}
\end{aligned}
$$

The denominator is easily evaluated and, apart from the constant factor $P$, the numerator is just the partial derivative of the denominator with respect to 


$$
x \equiv\left(p E^{\prime} \cos \omega t_{0}\right) / k T=
$$

Evaluation of the integrals leads to

$$
\langle p\rangle=p\left[\operatorname{coth} x-\frac{1}{x}\right]
$$

which for small $x$ reduces to

$$
\langle p\rangle=\frac{p^{2} E^{\prime}}{3 k T} \cos \omega t=\frac{p^{2} E^{\prime}}{3 k T} \operatorname{Re} e^{+j \omega t} e^{+}
$$

The mean polarization per unit volume, $\vec{P}(t)$, is obtained by multiplying eq. (2-39) by the number of molecules per unit of volume, $N$, and averaging over the time of the last collision. With random collisions, the probability that the last collision occurred in the interval $[t-(\phi+d \phi), t-d]$ is $(1 / \tau) \exp (-\phi / \tau) d \phi$, where $T$ is the mean interval between collisions. Consequently, since $\vec{P}(t)$ is parallel to $\vec{E}^{\prime}$,

$$
\begin{aligned}
\vec{P}(t) & =\frac{N p \vec{E}^{2}}{3 k T} \frac{1}{\tau} \int_{0}^{\omega} \cos \omega(t-\omega) e^{-\phi / \tau} d \phi \\
& =\frac{N p^{2} \vec{E}^{\prime}}{3 k T} \operatorname{Re}\left[e^{+j \omega t} \int_{0}^{\infty} e^{-(1+j \omega t) \omega / \tau} d \phi / \tau\right] \\
& =\frac{N p}{3 k T} \operatorname{Re}\left[\frac{E^{\prime} e^{+j \omega t}}{1+j \omega \tau}\right] .
\end{aligned}
$$

Writing $\vec{P}(t)=\operatorname{ReP}^{+}{ }^{+j \omega t}$, equation $(2-3.9)$ yields 


$$
\vec{P}=\frac{N p^{2} / 3 k T}{1+j \omega T} \vec{E}
$$

To express the local field $\vec{E}$, at a dipole in terms of the macroscopic applied field $\vec{E}$, the generalized Lorentz-Lorenz relation

$$
\vec{E}^{\prime}=\vec{E}+K \vec{P} / E_{0}
$$

is again introduced. Substituting this expression into eq.

(2-41) and solving for $\vec{P}$ now yields

$$
\vec{F}(\omega)=\epsilon_{0} X(\omega) \vec{E}(\omega),
$$

where for the Debye dielectric medium the dynamic susceptibility function is given by

$$
\chi(\omega)=\frac{\chi(0)}{1+j \omega \tau^{\prime}}
$$

and where

$$
x(0)=\frac{1}{\epsilon_{0}}\left(\frac{N^{2}}{3 k T}\right)\left[1-\frac{K}{\epsilon_{0}}\left(\frac{N_{p}^{2}}{3 k T}\right)\right]^{-1},
$$




$$
T^{\prime}=T\left[1-\frac{K}{E_{0}}\left(\frac{N^{2}}{3 k T}\right)\right]^{-1}
$$

The Lorentz-Lorenz field correction therefore has the effect of enhancing the Debye static susceptibility constant $x(0)$ and of broadening the frequency response of the dielectric medium by increasing the effective value of $\tau$. For $K=0$ the consequences of this correction vanish.

In the case where the dielectric medium is composed of several species of polar molecules, eq. (2-41) is replaced by

$$
\vec{P}=\left(\sum_{n}^{\frac{N_{n}^{P_{n}}}{1+j \omega T}}\right) \frac{\vec{E}^{\prime}}{3 k T^{\prime}}
$$

and eqs. (2-44) and (2-45) are generalized to read:

$$
x(0)=\frac{1}{3 k T \epsilon_{0}}\left(\sum_{n} N_{n} P_{n}^{2}\right)\left[1-\frac{K}{3 k T \epsilon_{0}}\left(\sum_{n} N_{n} P_{n}^{2}\right)\right]^{-1}
$$

and

$$
\tau^{\prime}=\tau\left[1-\frac{K}{\operatorname{KKT} \epsilon_{q}}\left(\sum_{n} N_{n} P_{n}^{2}\right)\right]^{-1} .
$$

Eq. (2-43) remains unchanged except for these modifications.

A quantum mechanical derivation of the Debye dielectric susceptibility function provides the same result. The quantum 
mechanical derivation differs from the classical version only in that the permissible dipolar orientations are discrete rather than continuous. The average is now obtained by summing over the various quantum states rather than integrating over a sphere as in eq. (2-27). Either way,

$$
\epsilon(\omega)=\epsilon_{0}[1+\chi(\omega)]=\epsilon_{0}\left[1+\frac{\chi(0)}{1+j \omega \tau^{\prime}}\right]
$$

21 
3. PLANE WAVE PROPAGATION AND REFLECTION

The set of four Maxwel 1 equations governing the electromagnetic field are:

$$
\begin{aligned}
& \nabla \times \vec{E}=-\frac{\partial \vec{B}}{\partial t}, \\
& \nabla \cdot \vec{H}=\frac{\partial \vec{D}}{\partial t}+\vec{J}, \\
& \nabla \cdot \vec{B}=0, \\
& \nabla \cdot \vec{D}=0 .
\end{aligned}
$$

For a linear medium containing oscillatory sources and fields these expressions simplify considerably. With the aid of constitutive relations $(2-5),(2-7),(2-8)$, and $(2-9)$, and for a sinusoidal field dependence such as given by eq. (2-13), Maxwel1"s equations reduce to:

$$
\begin{aligned}
& \nabla \times \vec{E}(\omega)=-j \omega \mu \vec{H}(\omega), \\
& \nabla \times \vec{H}(\omega)=(\sigma+j \omega \epsilon) \vec{E}(\omega)=j \omega \epsilon \operatorname{eff} \vec{E}(\omega),
\end{aligned}
$$




$$
\nabla \cdot \mu \vec{H}(\omega)=0,
$$

$$
\nabla \cdot \epsilon \vec{E}(\omega)=\rho(\omega) .
$$

If the medium is also homogeneous, and if the net free charge is everywhere zero $(0=0)$, then the 1 ast two equations become

$$
\begin{aligned}
& \nabla \cdot \vec{H}=0, \\
& \nabla \cdot \vec{E}=0 .
\end{aligned}
$$

Under the stated conditions, viz., harmonic oscillation and homogeneous, linear, charge-free space, the last pair of equations is redundant with the contents of the first two Maxwell equations. This is a consequence of a general theorem which states that the divergence of any vector, which is itself the curl of another vector, is identically zero. Accordingly, the divergence of eq. (3-5) or eq. (3-6) leads, respectively, to eq. $(3-9)$ or eq. $(3-10)$. On the other hand, the curl of eq. (3--5), upon substitution of eq. $(3-6)$ for $\nabla \times \vec{H}(\omega)$, gives:

$$
\nabla \times[\nabla \times \vec{E}(\omega)]-\omega^{2} \mu \epsilon \text { eff } \vec{E}(\omega)=0 .
$$

To investigate the properties of plane wave propagation, solutions to eq. (3-11) having the form 


$$
\vec{E}(\omega)=\vec{E}_{\omega} e^{-j \vec{k}(\omega) \cdot \vec{r}}
$$

are considered, where the vector constant $\vec{E}_{\omega}$ represents the amplitude and direction of the oscillating field intensity at frequency $\omega$. When this expression is combined with the time dependence of eq. (2-13) the complete space-time structure of the solution becomes evident; namely,

$$
\vec{E}(t)=\operatorname{Re} \vec{E}_{\omega} e^{j[\omega t-\vec{k}(\omega) \cdot \vec{r}]}
$$

When $\vec{k}(\omega)$ is real, such solutions propagate with velocity $\omega / k$ in the direction of $\vec{k}$ while maintaining a constant peak amplitude given by the magnitude of $\vec{E}_{\omega}$. Furthermore, since the phase of $\vec{E}(t)$ remains constant over a plane perpendicular to $\vec{k}$, this wave is planar. The dispersion relation for plane wave propagation is obtained by inserting eq. (3-12) into eq. (3-11):

$$
\vec{k} \times\left(\vec{k} \times \vec{E}_{\omega}\right)+\omega \epsilon_{e f f} \vec{E}_{\omega}=0 .
$$

Using a vector identity, this relation can also be written as:

$$
\left(\vec{k} \cdot \vec{E}_{\omega}\right) \vec{k}+\left(\omega^{2} \mu \epsilon f_{f}-k^{2}\right) \vec{E}_{\omega}=0
$$

For any given wave polarization and propagation direction this dispersion relation provides the magnitude of $\vec{k}$ and its dependence on frequency. From eqs. $(3-10)$ and $(3-12)$, or from 
the scalar product of $\vec{k}$ with eq. (3-14), it is evident that

$$
\vec{k}_{\vec{E}}=0
$$

The plane wave is consequently always polarized transverse to the direction of its propagation, and eq. (3-15) reduces to

$$
\left(\omega^{2} \mu \epsilon \operatorname{eff}^{\left.-k^{2}\right) \vec{E}_{\omega}}=0\right.
$$

Since the presence of radiation implies $\vec{E}_{\omega}$ is not zero, the quantity within the bracket must vanish. Evidently, plane wave propagation is supported only if the fields are transversely polarized and obey the 1 aw of dispersion:

$$
k(\omega)= \pm \omega \sqrt{\mu_{0} \epsilon_{\text {eff }}(\omega)} .
$$

The magnetic field intensity associated with the plane wave is readily determined by applying Maxwell's equation (3-5) to the expression $(3-12)$ :

$$
\vec{H}(\omega)=\vec{H}_{\omega} e^{-j \vec{k}(\omega) \cdot \vec{r}}=\frac{\vec{k}}{\omega \mu_{0}} \times \vec{E}_{\omega} e^{-j \vec{k}(\omega) \cdot \vec{r}} .
$$

Therefore, at each location within the radiation, the ratio of the magnetic field intensity to the electric field intensity remains constant at the value $k / \omega \mu_{0}=\sqrt{\epsilon_{\text {eff }}(\omega) / \mu_{0}}$. Furthermore, the directions of the electric field, the magnetic field, and the propagation are always mutually orthogonal.

For plane wave propagation within an infinite isotropic and homogeneous space all transverse directions of wave polarizatior 
are equivalent. The presence of a surface will, however, break the symmetry of the space and eliminate the degeneracy present in the wave polarization. A preferred "plane of incidence" is defined by the unit vector, $\hat{n}$, normal to the surface and the propagation vector, $\vec{k}$. Relative to this plane an arbitarily polarized plane wave can be resolved into two fundamentally different, orthogonally polarized, plane wave components (figure 1). The plane wave component polarized perpendicular to the plane of incidence is said to be s-polarized, or transverse electric (TE), and has as its field components:

$$
E_{\omega \perp}=(\hat{k \times n}) \cdot \vec{E}_{\omega} .
$$

$$
H_{\omega \perp}=\sqrt{\epsilon_{e f f} / \mu}[\hat{k} \times(\hat{k} \times \hat{n})] \cdot \vec{E}_{\omega}
$$

where $\hat{k}=\hat{k} / k$ is the unit vector in the direction of propagation. The other plane wave component, polarized parallel to the plane of incidence, is said to be p-polarized, or transverse magnetic (TM), and has as its field components

$$
E_{\omega \|}=[\hat{k} \times(\hat{k} \times \hat{n})] \cdot \vec{E}_{\omega}
$$

$$
H_{\omega \|}=-\sqrt{\epsilon_{\text {eff }} / \mu_{0}}(\hat{k} \times \hat{n}) \cdot \vec{E}_{\omega}
$$

At a planar boundary an incident wave of either polarization generates both reflected and transmitted waves of the same polarization. The amplitudes and directions of the reflected and 

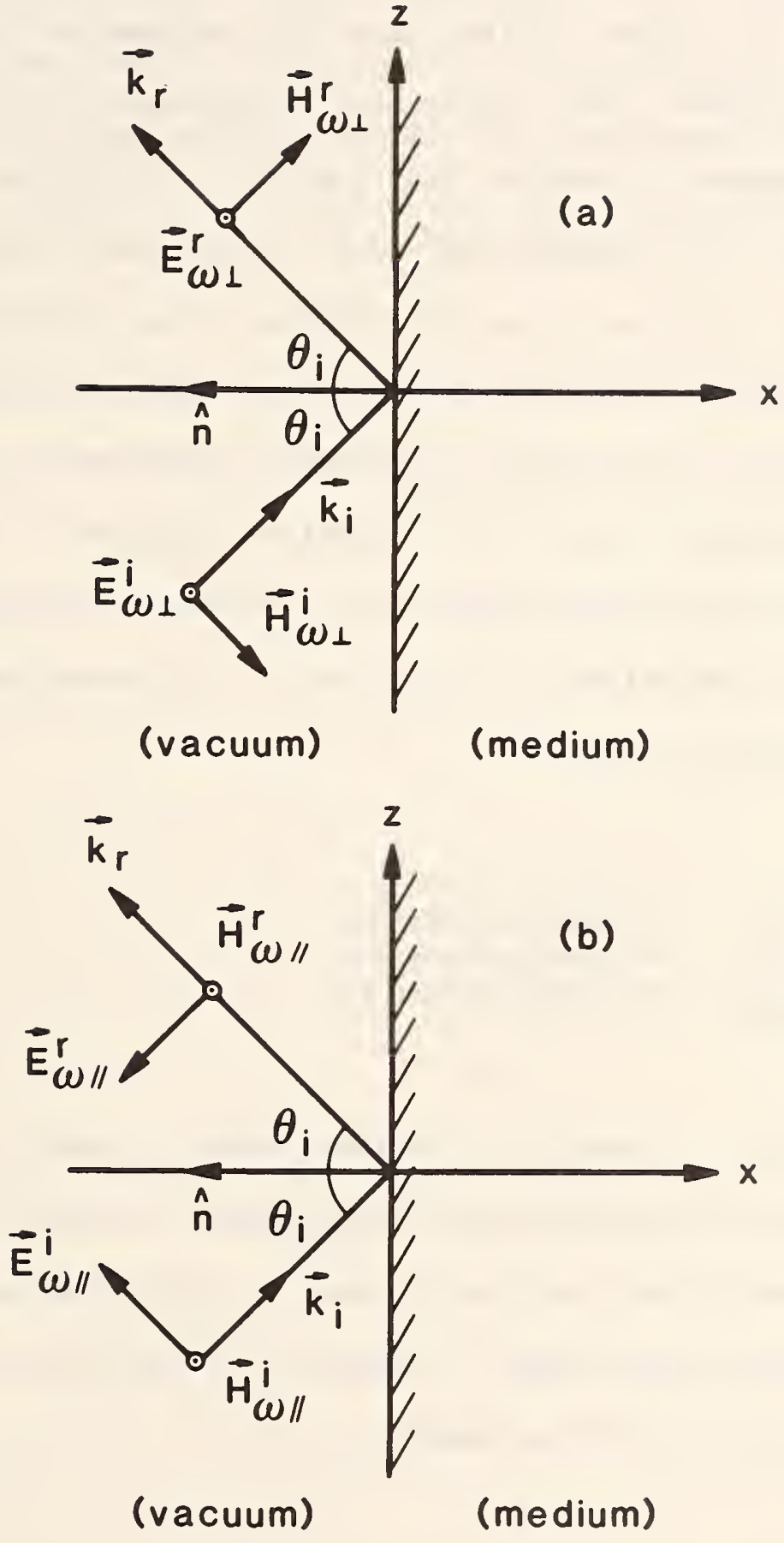

Figure 1. Reflection of a planewave at a material half-space. (a) Field vectors for the transverse electric (TE) polarized component. (b) Field vectors for the transverse magnetic (TM) polarized component. 
transmitted plane wave fields are determined not only by the incident wave, but also by the material parameters at the boundary. In general, the tangential components of the total electric and magnetic fields must each be continuous across a surface. Since the s-polarized plane wave has only a tangential electric field, and the p-polarized plane wave has only a tangential magnetic field, the two forms satisfy different boundary conditions and are reflected independently from the surface. A different law of reflection applies in each case. For the TE-polarized plane wave the (Fresnel) reflection coefficient for the electric field at a vacuum-dielectric boundary is given by [16]:

$$
R_{s}(\omega)=\frac{E_{\omega \perp}^{r}}{E_{\omega \perp}^{i}}=\frac{1-\sqrt{1+\chi(\omega) / \cos ^{2} \theta} i}{1+\sqrt{1+\chi(\omega) / \cos ^{2} \theta_{i}}} .
$$

The superscripts "i" and "r" respectively, refer to the incident and reflected wave components of the total field, and $\theta_{i}$ is the angle of incidence defined in figure 1. For the corresponding TM-polarized plane wave the (Fresnel) reflection coefficient for the magnetic field at this boundary is:

$$
R_{p}(\omega)=\frac{H_{\omega \|}^{r}}{H_{\omega \|}^{i}}=\frac{[1+\chi(\omega)]-\sqrt{1+\chi(\omega) / \cos ^{2} \theta_{i}}}{[1+\chi(\omega)]+\sqrt{1+\chi(\omega) / \cos ^{2} \theta_{i}}} .
$$


At normal incidence, when the angle of incidence $\theta_{i}$ is zero and $R_{s}=-R_{p}$. As expected, both reflection coefficients reduce to the same expression because a plane of incidence can no longer be defined. The minus sign is merely a consequence of the conventions adopted in figure 1 for the positive field directions. 
4. INTEGRAL TRANSFORMATIONS AND THE FRESNEL THEORY

Classical fresnel theory treats the reflection and transmission of plane waves at flat, non-dispersive surfaces. The generalization of this theory to include non-monochromatic waveforms and dispersive media is readily accomplished by introducing Fourier analysis methods. By means of the Fourier transformation integral

$$
\vec{E}^{i}(\omega)=\int_{-\infty}^{\infty} \vec{E}^{i}(t) e^{-j \omega t} d t,
$$

and its inverse

$$
\vec{E}^{i}(t)=\frac{1}{2 \pi} \int_{-\infty}^{\infty} \vec{E}^{i}(\omega) e^{+j \omega t} d \omega,
$$

an incident plane wave, having arbitarily specified waveform $\vec{E}^{i}(t)$ at the reflecting surface is resolved into a spectrum of truly monochromatic plane waves, each of (real) frequency $\omega$ and spectral amplitude $\vec{E}^{i}(\omega)$. Furthermore, if the reflecting medium is assumed to respond linearly, each spectral component may be treated independently using the Fresnel reflection coefficient $R(w)$ appropriate to the frequency and polarization of that component:

$$
\vec{E}^{r}(\omega)=R(\omega) \vec{E}^{i}(\omega)
$$

The reflection of the actual incident plane wave is subsequently determined by superimposing the individually reflected spectral 
components via the inversion integral:

$$
\vec{E}^{r}(t)=\frac{1}{2 \pi} \int_{-\infty}^{\infty} \vec{E}^{r}(\omega) e^{+j \omega t} d \omega=\frac{1}{2 \pi} \int_{-\infty}^{\infty} F(\omega) \vec{E}^{i}(\omega) e^{+j \omega t} d \omega \cdot(4-4)
$$

For the transverse-electric polarized plane wave the Fresnel reflection coefficient is given by eq. (3-24). A completely analogous expression in terms of the magnetic field can be written for the transverse-magnetic polarized plane wave, with $R(\omega)$ given by eq. $(3-25)$.

A further generalization of the Fresnel theory is achieved if, instead of the Fourier transform, the Laplace transform is employed. The Laplace transform facilitates the investigation of transient phenomena initiated by the sudden arrival (at time $t=0$ ) of a wave at the reflecting surface. The Laplace transform of $E^{i}(t)$ is defined by the pair of integrals:

$$
\vec{E}^{i}(s)=\int_{0}^{\infty} \vec{E}^{i}(t) e^{-s t} d t
$$

and

$$
\vec{E}^{i}(t)=\frac{1}{2 \pi j} \int_{\gamma-j \infty}^{\gamma+j \infty} \vec{E}^{i}(s) e^{+s t} d s .
$$

Transform variable $s=\sigma+j \omega$ is generally complex as variables $\sigma$ and $w$ are both real. The number $\gamma$, appearing as part of the integration 1 imits of eq. (4-6), is also real and is chosen so that the integration path $\sigma=\gamma$ in the plane of complex frequencies 
$s$ lies to the right of all singularities of $\vec{E}^{i}(s)$. The bounded nature of any physical field at infinite times assures, however, that all singularities of $\vec{E}^{i}$ (s) occur in the left half-plane where Re 5\$O. So, for all practical purposes, $\gamma$ approaches zero as its minimum limit.

A useful interpretation of the Laplace transform is to regard integral (4-6) as a superposition of exponentially growing and decaying sinusoidal components which combine to form $\vec{E}^{i}(t)$. The amplitudes $\vec{E}^{i}$ (s) of these components are resolved using integral (4-5). The Fourier transform and the Laplace transform are very closely related. In fact, a formal conversion of Fourier transform pair (4-1) and (4-2) into Laplace transform pair (4-5) and (4-6) is readily achieved by substituting 5 for jw and recognizing that $\vec{E}^{i}(t)$ is zero until the incident wave arrives at the surface at time $t=0$. This same substitution analytically continues $R(\omega)$ into the reflection coefficient $R(s)$ for the spectral component $\vec{E}^{i}(s)$. By analogy to eq. (4-4) the entire reflected wave at the surface is given by

$$
\vec{E}^{r}(t)=\frac{1}{2 \pi j} \int_{\gamma-j \infty}^{\gamma+j \infty} \vec{E}^{r}(s) e^{+s t} d s=\frac{1}{2 \pi j} \int_{\gamma-j \infty}^{\gamma+j \infty} R(s) \vec{E}^{i}(s) e^{+s t} d s .
$$

The principle of causality prevents the reflection $E^{r}(t)$ from initiating before the arrival of the incident wave at $t=0$. This observation may be applied to eq. (4-7) to demonstrate that neither $\vec{E}^{r}$ (s) nor $R(s)$ can have any singularities in the right half of the complex $s$ plane. 3 The reflection coefficient for 
treating TE plane wave transients is given by the analytical continuation of eq. $(3-24)$,

$$
R(5)=\frac{1-\sqrt{1+\chi(5) / \cos ^{2} \theta} i}{1+\sqrt{1+X(5) / \cos ^{2} \theta}}
$$

where the dielectric susceptibility $\chi(5)$ is some rational or otherwise well-behaved function of 5 having no singular points in the right half plane.

The inverse Laplace transform of $R(s)$, given by the timedependent function

$$
R(t)=\frac{1}{2 \pi j} \int_{\gamma-j \infty}^{\gamma+j \infty} F(s) e^{+s t} d s,
$$

has a simple and useful physical interpretation. Consider an incident plane wave which has as its waveform a Dirac delta "function" profile of unit amplitude:

$$
E^{i}(t, \vec{r})=\delta\left(t-\frac{x}{c} \cos \theta_{i}-\frac{z}{c} \sin \theta_{i}\right) .
$$

3 Suppose the path of integration for eq. (4-7) is closed with an arc of infinite radius surrounding the right half plane of $s$ where $\sigma$ is positive. For $t<0$, integration along this arc contributes nothing to integral (4-7) because the integrand exponentially vanishes here. On the other hand, the Cauchy residue theorem, together with the causal condition $\hat{E}^{\prime}(t=0)$ for $t<0$, guarantee that no singularities of either $E^{\prime}(5)$ or $R(5) E^{1}(5)$ occur within the enclosing contour of inţegration, i.e., the right half plane. Furthermore, because $\mathrm{E}^{1}$ (s) has no singularities in this portion of the complex frequency plane then neither has $\mathrm{R}(\mathrm{s})$. 
At some point $z=0$ on the surface $x=0$ the Laplace transform of this waveform is

$$
E^{i}(s)=\int_{0}^{\infty} \delta(t) e^{-s t} d t=1
$$

According to eq. (4-7) the amplitude of the reflected waveform at this location is

$$
E^{r}(t)=\frac{1}{2 \pi j} \int_{\gamma-j \infty}^{\gamma+j \infty} R(s) e^{+s t} d s=R(t) .
$$

Thus $R(t)$ describes the temporal response of the surface to an impulsive waveform of unit amplitude and is named the imgulls

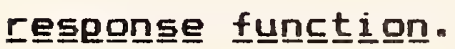

For observation points off the surface the reflected wave must display spatial as well as temporal dependence. Generalization of $R(t)$ is accomplished by recognizing that upon reflection the normal component of an incident wave's propagation vector is reversed. Consequently, the reflection of plane wave (4-10) is readily constructed from $R(t)$ by substituting for $t$ the

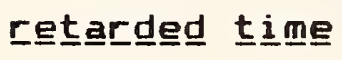

$$
[t]=t+\left(x \cos \theta_{i}-z \sin \theta_{i}\right) / c .
$$

The generalized impulse response function R[t] is referred to as

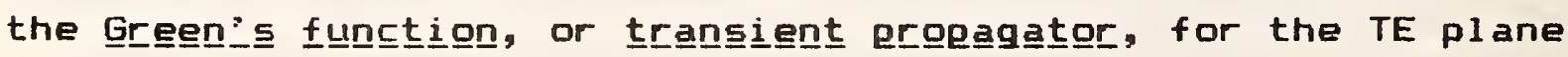
wave reflection problem. 
To be worthy of its title fit] must completely characterize the elementary reflection process at any given observation point and at any particular instant of time. To demonstrate this property of R[t] observe that according to eq. (4-7), for any incident plane wave described on the surface by transform $\vec{E}^{i}$ (s), the reflected plane wave anywhere in front of the surface is given by

$$
\vec{E}^{r}(t, \vec{r})=\vec{E}^{r}[t]=\frac{1}{2 \pi j} \int_{\gamma-j \infty}^{\gamma+j \infty} R(s) \vec{E}^{i}(s) e^{+s[t]} d s .
$$

Substitution of definition (4-5) for $\vec{E}^{i}$ (5) and the corresponding integral for $R(s)$ and rearrangement of the integrations permits eq. (4-14) to be converted into the following convolution integral:

$$
\vec{E}^{r}(t, \vec{r})=\int_{0}^{t]} \vec{E}^{i}(\tau) R([t]-\tau) d \tau
$$

By means of expression (4-15) the transient reflection of any TE plane waveform specified by $\vec{E}^{i}(t)$ can be computed once $R[t]$ is in hand.

Integral (4-15) can be viewed as a superposition describing the total reflection caused by a train of impulsive plane waves. Thus, if the amplitude of the incident waveform appearing on the surface within the infinitesimal time span $t=\tau$ to $t=\tau+\Delta \tau$ is viewed as a Dirac delta function of strength $\vec{E}^{i}(\tau) \Delta \tau$, then $\vec{E}^{i}(\tau) \Delta \tau R([t]-\tau)$ is the amplitude of its reflection observed to commence at location $(x, z)$ at later time $t=\tau-\left(x \sin \theta_{i}-z \cos \theta_{i}\right) / c$. 
Furthermore, the complete waveform $\vec{E}^{r}(t, r)$ is seen to consist of a superposition of such elementary reflections from all earlier times, i.e., from $t=0$ until the present,

$$
\vec{E}^{r}(t, \vec{r})=\vec{E}^{i}(0) \Delta \tau R[t]+\cdots
$$

$+\vec{E}^{i}(\tau) \Delta \tau R([t]-\tau)+\ldots+\vec{E}^{i}[t] \Delta \tau R[0]$

$$
=\sum_{n=0}^{m} \vec{E}^{i}(n \Delta \tau) R([t]-n \Delta \tau) \Delta \tau .
$$

Equation (4-15) is recovered in passing to the 1 imit in which $m \equiv[t] / \Delta \tau \rightarrow \infty$ as $\Delta \tau \rightarrow O$, while $m \Delta \tau=[t]$ remains $f i x e d$. 


\section{CONGTRUCTION OF THE TRANSIENT PROPAGATOR}

An algorithm will now be presented for constructing the transient propagator describing TE plane wave reflections into vacuum. The reflecting medium is assumed to be a non-magnetic dielectric half-space modeled by a complex susceptibility function, $\chi(j \omega)$. If the reflecting medium has any conductivity it is included in $X(j \omega)$. Under these circumstances the conventional Fresnel reflection coefficient, originally presented as eq. $(3-24)$, applies:

$$
F(j \omega)=\frac{1-\sqrt{1+\bar{\chi}(j \omega)}}{1+\sqrt{1+\bar{\chi}(j \omega)}}
$$

where

$$
\bar{x}(j \omega)=x(j \omega) / \cos ^{2} \theta_{i}
$$

To facilitate computations, the function $R(j \omega)$ is rationalized by multiplying both numerator and denominator by $1-\sqrt{1+\bar{X}(j \omega)}$. This leads to the expression:

$$
R(j \omega)=-1+\frac{2}{\bar{x}(j \omega)}[-1+\sqrt{1+\bar{x}(j \omega)}] .
$$

The radical remaining in expression (5-3) is eliminated by expanding it as a binomial series: 


$$
R(j \omega)=\frac{1}{4} \sum_{n=0}^{\infty} \frac{(3 / 2) n}{(3)_{n}}[-\bar{x}(j \omega)]^{n+1} .
$$

Appearing within the summation is the Pochhammer symbol defined by

$$
(z)_{n}=\Gamma(z+n) / \Gamma(z)=z(z+1)(z+2) \ldots(z+n-1)
$$

$\Gamma(z)$ is the gamma function, which among its many properties, is best known as the generalization of the factorial function to non-integer and generally complex numbers.

To address the transient problem, reflection coefficient (5-4) is analytically continued into the complex frequency plane by replacing $j \omega$ by the Laplace transform variable s:

$$
R(s)=\frac{1}{4} \sum_{n=0}^{\infty} \frac{(3 / 2) n}{(3)_{n}}[-\bar{x}(s)]^{n+1}
$$

As eq. (5-6) stands, $R(5)$ is expressed as a summation of rational functions in the variable 5 . Term by term inversion of $R$ (s) in this form can now be readily accomplished. In certain cases it will be possible to sum the resulting time series for $R(t)$. In other cases the impulse response function must be approximated by considering an appropriate number of terms in the series.

Construction of the transient propagator is completed by replacing the argument of $R(t)$ by the retarded time [t]. 
According to eq. (4-13),

$$
[t]=t+\frac{x}{c} \cos _{i}-\frac{z}{c} \sin \theta_{i}=
$$

$(5-7)$

Once Rit] has been determined for a given material it may be used in conjunction with convolution integral (4-15) to predict the reflection of any specified, incident, plane waveform. The remainder of this report is devoted to applying this algorithm to the dispersive materials modeled in chapter 2.

39 
6. THE TRANSIENT PROPAGATOR FOR TREATING REFLECTIONS FROM A LORENTZ DIELECTRIC

The frequency-dependent susceptibility function for the Lorentz dielectric model was presented in eq. (2-29) for a material composed of several different kinds of oscillators. In the present discussion the Lorentz dielectric will simply consist of a single species of oscillators, viz,, electrons bound to their atomic cores with harmonic frequency $\omega_{0}$ (binding energy $\left.k \omega_{0}\right) .4$ For this case eq. $(2-29)$ reduces to

$$
x(j \omega)=\frac{\omega_{p}^{2}}{\left(\omega_{0}^{2}-\omega^{2}\right)+j \omega \nu} .
$$

As earlier, the strength of the collective interaction between the oscillators and the electromagnetic field is represented by the second power of the plasma frequency $\omega_{p}$. This quantity is proportional to the average charge density of the oscillator electrons. The oscillator motion is also subject to a viscous damping force characterized by the collision frequency, $v$. The latter manifests itself either as a 1 inewidth in a spectral measurement, or as a reciprocal 1 ifetime in a relaxation measurement.

To construct the transient propagator R[t] the dielectric susceptibility function $X(j \omega)$ is analytically continued from the

4 Due to corrections, such as given by eq. (2-28), accounting for the presence of neighboring oscillators, $w_{0}$ is not precisely the binding frequency. 
imaginary axis into the plane of complex frequencies by replacing jw by 5 in eq. $(6-1)$.

$$
x(s)=\frac{\omega_{p}^{2}}{s^{2}+\nu \omega_{0} \omega_{0}^{2}}=\frac{\omega_{p}^{2}}{\left(5-s_{1}\right)\left(5-s_{2}\right)} .
$$

The poles of $X(5)$ located at $5_{1}$ and $s_{2}$ are observed to be real when $\nu \geqslant 2 \omega_{0}$, or el se complex conjugates when $v<2 \omega_{0}$ :

$$
s_{1}, s_{2}= \begin{cases}-\frac{\nu}{2} \pm \sqrt{\left(\frac{\nu}{2}\right)^{2}-\omega_{0}^{2}} & , \text { for } v>2 \omega_{0} \\ -\frac{\nu}{2}=-\omega_{0} & , \text { for } \nu=2 \omega_{0} \\ -\frac{\nu}{2} \pm j \sqrt{\omega_{0}^{2}-\left(\frac{\nu}{2}\right)^{2}} & \text {, for } v<2 \omega_{0} .\end{cases}
$$

The temporal response of the dielectric is determined by the poles of its susceptibility function. For example, the inverse Laplace transform associated with a simple pole is an exponential having this pole as its characteristic frequency,

$$
L^{-1}\left[\frac{A}{(s-5 a)}\right]=A e^{s t} .
$$

Referring to eq. (2-15), the poles of the oscillator displacement function $r(5)$ are seen to be identical to those of $x(5)$.

Therefore, the dielectrical behavior of the medium as $v$ is varied can be directly interpreted in terms of the oscillator motion. 
The locus of the poles in the complex splane, as $\nu$ is increased from zero through infinite values, is shown in figure 2. For $\nu=0$ the poles are located on the imaginary axis at $5= \pm j \omega_{0}$ and the oscillator electrons vibrate with resonant frequency $\omega_{0}{ }^{\circ}$ As $v$ ranges from zero to $\nu=2 \omega$, the poles move as complex conjugates in the left half plane along a semi-circular trajectory of radius "o centered at the origin. Within this range the electronic motion is underdamped, i.e., it is oscillatory but exponentially attenuated with increasing time. For $\nu=2 w_{0}$, the poles coalesce on the real axis at $s=-\nu / 2=-\omega_{0}$. Now $r(t)$ is critically damped, reflecting a transition to nonoscillatory motion. For $\nu>2 \omega_{0}$ the motion is heavily damped and characterized by two real time constants; one appraaching zero, the other approaching infinity, as $\nu+\infty$. The pole locus therefore defines three distinct regimes for investigating reflections from simple Lorentz dielectrics. These regimes correspond to dielectric media which are underdamped ( $\left.\nu<2 \omega{ }_{0}\right)$, critically damped $\left(\nu=2 \omega_{0}\right)$, and overdamped $\left(\nu>2 \omega_{0}\right)$.

To continue with the construction it is necessary to identify $\bar{x}(5)$ in eq. (5-6). According to eq. (5-2),

$$
\bar{x}(s)=x(s) / \cos ^{2} \theta_{i}=\frac{\bar{\omega}_{p}^{2}}{\left(5-s_{1}\right)\left(5-s_{2}\right)}
$$

where $\bar{\omega}_{p} \equiv \omega_{p} / \cos \theta_{i}$. Therefore 


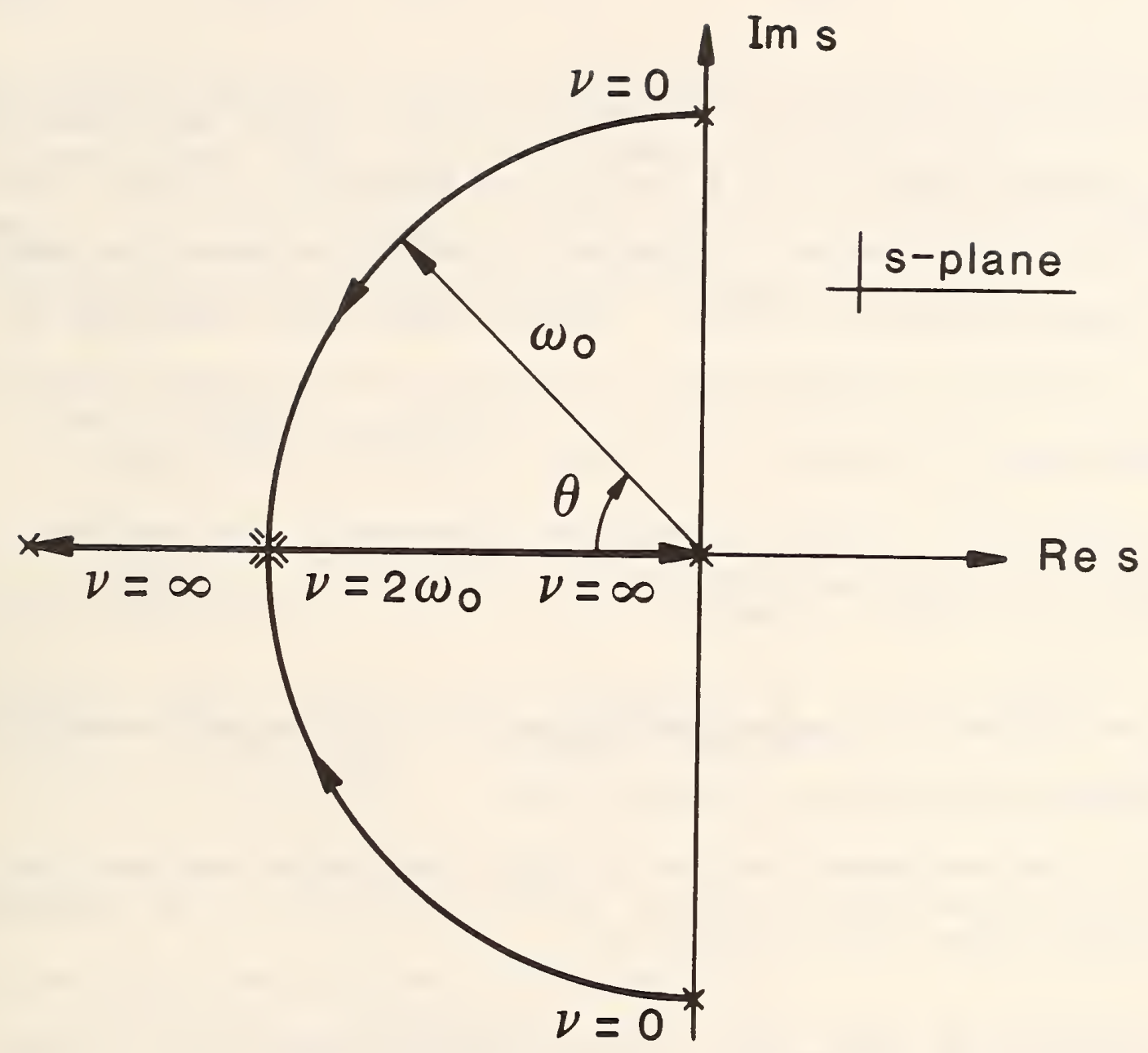

Figure 2. The locus traced by the poles of $\chi(s)$ as the inelastic collision frequency $\nu$ is varied between zero and infinity. For $0 \leqslant v \leqslant 2 \omega$, the roots follow a circular locus of radius $\omega$ with $\theta= \pm \cos \left(v / 2 \omega{ }^{\prime}\right)$. For $v>2 \omega$, the locus coincides with the negative half of the real axis. 


$$
R(5)=-\frac{1}{4}\left(\bar{\omega}_{p}\right)^{2} \sum_{n=0}^{\infty}(-1)^{n} \frac{(3 / 2) n}{(3)_{n}} \frac{\left(\bar{\omega}_{p}\right)^{2 n}}{\left(5-5_{1}\right)^{n+1}(5-5)^{n+1}}
$$

Note that the simple poles of $r(s)$ and $X(s)$ appear as simple and higher order poles in the power series expansion of $R(s)$. As a result $R(t)$ will contain terms more complicated than the exponential functions given by eq. (b-4). Nevertheless, the inversion of $R(5)$ is readily accomplished by applying the following Laplace transform pair (A+S 29.3 .50$)^{5}$ term-by-term to eq. (b-6):

$$
\frac{\Gamma(k)}{(s+a)^{k}(s+b)^{k}} \underset{(k>0)}{\stackrel{2}{\pi}}\left(\frac{t}{a-b}\right)^{k-1 / 2} e^{-(a+b) t / 2} I_{k-1 / 2}\left(\frac{a-b}{2} t\right) \cdot(6-7)
$$

The 1 ast factor is a modified Bessel function of the first kind, of argument $(a-b) t / 2$ and order $k-1 / 2$.

In the overdamped regime $\left(\nu>2 \omega_{0}\right)$, the poles are real and, according to eq. (b-3), given by

$$
s_{1}, s_{2}=-\frac{\nu}{2} \pm \alpha, \text { where } \alpha \equiv \sqrt{(\nu / 2)^{2}-\omega_{0}^{2}} .
$$

The corresponding impulse response function. is determined by applying transformation (6-7) to expression (6-6). Since $n$ is ari integer, $\Gamma(n+1)=n !$ and

5 Parenthetical entries of this form refer to formulas tabulated in the Handbook of Mathematical Functions, M. Abramowitz and I. A. Stegun; c.f. References at conclusion of this report. 


$$
\frac{R(t)}{\bar{\omega}_{p}}=-\frac{1}{4}\left(\bar{\omega}_{p} t\right)\left\{\sum_{n=0}^{\infty}(-1)^{n} \frac{(3 / 2) n}{(3) n^{n !}}\left[\frac{\left(\omega_{p} t\right)}{2 \alpha t}\right]^{n} \frac{\pi}{2 \alpha t} I_{n+1 / 2}(\alpha t)\right\} e^{-\nu t / 2} .
$$

The impulse response function consequently appears as a summation of products each consisting of a numerical coefficient, a monomial, a modified Bessel function, and an exponential factor. By introducing the modified spherical Bessel function:

$$
i_{n}(\alpha t) \equiv \sqrt{\frac{\pi}{2 \alpha t}} I_{n+1 / 2}(\alpha t)
$$

eq. (6-9), for the overdamped dielectric ( $\left./ 2 \omega_{0}\right)$, is simplified.

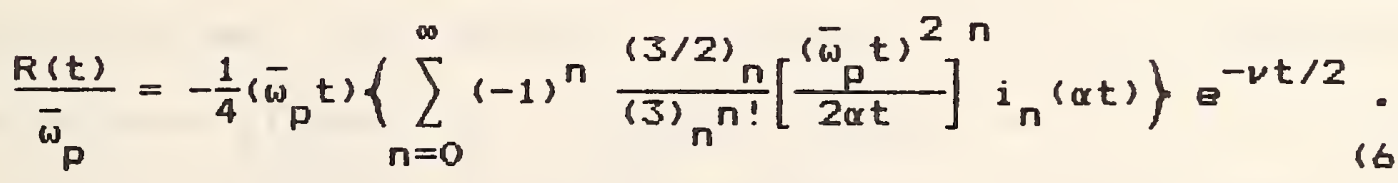

Expression (6-11) is dimensionless because, as in eq. (4-15),

$R(t)$ has the dimensions of reciprocal time.

In the underdamped regime $\left(\nu<2 \omega_{0}\right)$, the poles are complex conjugates and, according to eq. (b-3), given by

$$
s_{1}, s_{2}=-\frac{\nu}{2} \pm j \omega_{c} \text {, where } \omega_{c} \equiv \sqrt{\omega_{0}^{2}-(\nu / 2)^{2}}=j \alpha \text {. }
$$

Comparison of eq. (6-8) with eq. (6-12) shows that result (6-11) can be adapted to the underdamped dielectric case by replacing $\alpha$ by $-j \omega c \cdot$ Since 


$$
i_{n}(\alpha t)=i_{n}\left(-j \omega_{c} t\right)=(-j)^{n} j_{n}\left(\omega_{c} t\right) \text {, }
$$

where $j_{n}\left(\omega_{c} t\right)$ is a spherical Bessel function of the first kind, the impulse response function for the underdamped dielectric $\left(\nu<2 \omega_{0}\right)$ can be written as

$$
\frac{R(t)}{\bar{\omega}_{p}}=-\frac{1}{4}\left(\bar{\omega}_{p} t\right)\left\{\sum_{n=0}^{\infty}(-1)^{n} \frac{(3 / 2) n}{(3) n^{n !}}\left[\frac{\left(\bar{\omega}_{p} t\right)^{2}}{2 \omega_{c} t}\right]^{n} j_{n}\left(\omega_{c} t\right)\right\} e^{-v t / 2} .
$$

Expressions $(6-11)$ and $(6-14)$ describing the reflection of an impulsive field are reminscient of a modal expansion; the higher order modes corresponding to higher orders of the simple poles appearing in $r(s)$ and $X(5)$. Furthermore, the appearance of $i_{n}(\alpha t)$ in $(b-11)$, or $j_{n}\left(\omega_{c} t\right)$ in $(6-14)$, is readily associated with, respectively, the monotonic decay in the motion of an overdamped oscillator, or the vibratory decay of an underdamped oscill 11 ator.

In the critically damped regime $\left(\nu=2 \omega_{0}\right)$, the Lorentz dielectric medium is characterized by a real, second order pole at $s=-\nu / 2=-\omega_{0}$, corresponding to $\alpha=\omega_{c}=0$. For $5 m a 11$ arguments, the 1 imiting value of the spherical Bessel function $(A+510.1 .4,6.1 .12$, and 6.1 .22$)$ is given by 


$$
j_{n}\left(\omega_{c} t\right) \rightarrow \frac{\left(\omega_{c} t\right)^{n}}{2^{n}(3 / 2)_{n}}
$$

With this substitution, eq. (6-14) reduces to

$$
\frac{R(t)}{\bar{\omega}_{p}}=-\frac{1}{2}\left(\frac{\bar{\omega}_{p} t}{2}\right)\left\{\sum_{n=0}^{\infty} \frac{1}{(3)_{n} !}\left[-\left(\frac{\bar{\omega}_{p} t}{2}\right)^{2}\right]^{n}\right\} e^{-\nu t / 2} .
$$

The quantity within the curly brackets is recognized as being the defining series for the hypergeometric function ${ }_{0} F_{1}\left[3 ;-\left(\omega_{p}^{\prime} t / 2\right)^{2}\right]$, so that

$$
\frac{R(t)}{\bar{\omega}_{p}}=-\frac{1}{2}\left(\frac{\bar{\omega}_{p} t}{2}\right)_{0} F_{1}\left[3 ;-\left(\frac{\bar{\omega}_{p}}{2}\right)^{2}\right] e^{-\nu t / 2} .
$$

Use of the identity (A+5 9.1.69):

$$
J_{n}(z)=\frac{1}{\Gamma(n+1)}\left(\frac{z}{2}\right)^{n}{ }_{0} F_{1}\left[n+1 ;-\left(\frac{z}{2}\right)^{2}\right]
$$

permits eq. (6-17) to be re-expressed in terms of Bessel function $J_{2}\left(\omega_{p}^{\prime} t\right)$.

$$
\frac{R(t)}{\bar{\omega}_{p}}=\frac{-2}{\left(\bar{\omega}_{p} t\right)} J_{2}\left(\bar{\omega}_{p} t\right) e^{-\nu t / 2} \text {. }
$$


Apparently, the coalescence of the two simple polas of $r(s)$ and $X(s)$ into a single second order pole in the critically damped case coincides with a corresrunding condensation of the modal expansion into a sing!e oscillatory term.

The tinal step in constructing a transient propagator R[t] requires the substitution of eq. (5-7) for $t$ in the impulse response functions $F(t)$. Namely, the substitution of

$$
[t]=t+\left(x \cos \theta_{i}-z \sin \theta_{i}\right) / c
$$

for $t$ in eqs. $(6-11),(6-14)$, and (b-19). The behavior of these transient propagators is illustrated as a function of the retarded time in figure 5 for a choice of parameters. At the point $(x=0, z=0)$ on the dielectric surface, the reflected pulse is first observed at $t$ ime $t \equiv[t]=0$. However, at a location situated in front of the dielectric surface, where $x$ is negative, the reflected pulse does not appear until $[t]=0$, i.e., not until time 't $=\left(1 \times 1 \cos \theta_{i}+2 \sin \theta_{i}\right) / c=$ 

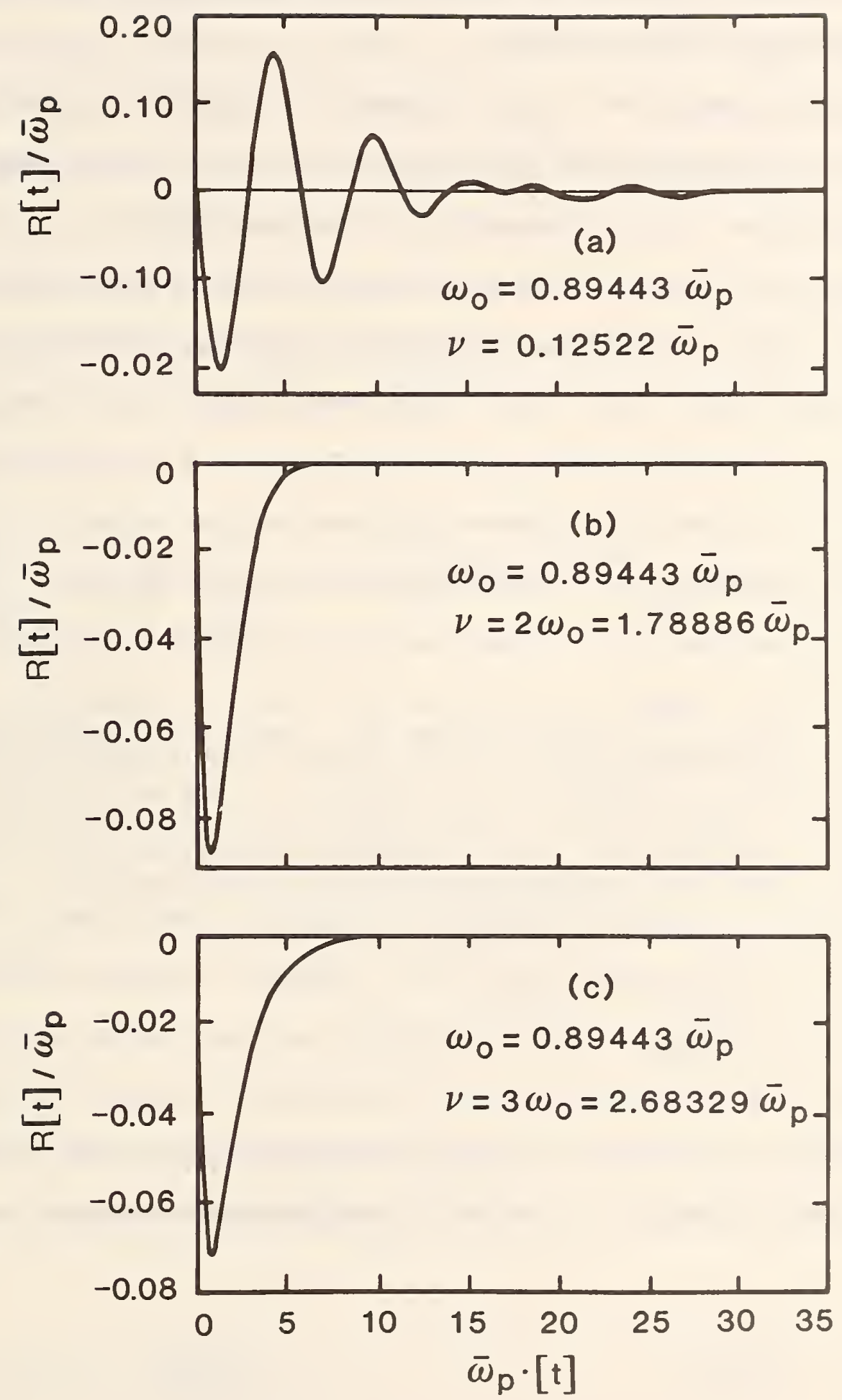

Figure 3. The transient propagator for treating reflections from a Lorentz dielectric half-space. (a) Underdamped medium, (b) Critically damped medium, (c) Dverdamped medilim. The parameters in case (a) were chosen to agree with those used by Sommerfeld and $\mathrm{Brill} l$ ouin [z]. 
7. THE TFANSIENT FROPAGATOR FOR TREATING REFLECTIONS FROM A DRUDE METAL AND A PLASMA

The Drude model of a metal describes electrical conduction in terms of the motion of an electron gas which undergoes momentum dissipating collisions with an ion lattice it freely permeates. The lattice functions to provide charge neutrality to the metal. Band structure, anisotropy, spatial dispersion, and other properties of the solid state are totally ignored. As a consequence, the mathematical description of a Drude metal is identical with that of a gaseous plasma to be found in the ionosphere, the sun, or a thermonuclear fusion device.

The properties of a Drude medium are readily deduced from the Lorentz model by setting the binding frequency ${ }_{0}$ of the dielectric ascillator electrons to zero. The susceptibility function which results from performing this operation is given by eq. (2-33), and for a single species of electrons is

$$
x(j \omega)=\frac{-\omega^{2}}{\omega(\omega-j \nu)} .
$$

The reduced susceptibility function appearing in eqs. (5-1) and (5-2), when expressed in terms of the complex frequency 5 , viz.,

$$
\bar{x}(s)=\frac{\bar{\omega}_{P}^{2}}{s(s+v)},
$$

has real poles at $s=0$ and $s=\nu$, with $\bar{\omega}_{p}=\omega_{p} / \cos \theta_{i}$. While one pole remains fixed at the origin, the other travels with increasing $v$ along the negative real axis from the origin to negative 
infinity. By having set $\omega_{0}$ to zero the semi-circular portion of the pole locus, shown in figure 2 for a Lorentz dielectric, has been collapsed to a point at the origin. The remaining portion of the pole locus, ascribed earlier to an gyergagmege Lorentz dielectric, now corresponds to the Drude metal. The impulse response function for the Drude metal is therefore most easily determined by placing $\omega_{0}=0$ in expression (6-11). In that case, according to eq. $(6-B), \alpha=\nu / 2$ and

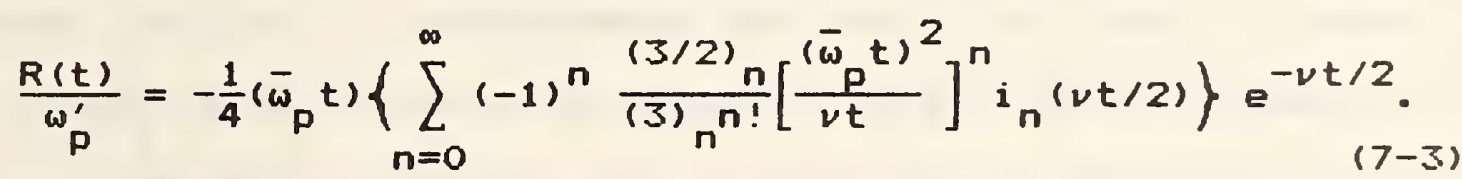

As already mentioned, the description of a cold, collisionless plasma as a tenuous electrically neutral gas of electrons and positively charged ions is identical to the Drude model of a metal when $\nu=0$ and the motion of the much more massive positive ions can be ignored. For $\nu=0$, both poles of $X(5)$ are fixed at the origin of the complex frequency plane. The impulse response function for the plasma is determined by letting $\nu+0$ in eq. (7-3). In this $1 \mathrm{imit}$ the exponential approaches unity and the Bessel function approaches $(A+510.2 .5,6.1 .12$, and 6.1 .22$)$

$$
i_{n}(\nu t / 2)+\frac{(\nu t / 2)^{n}}{2^{n}(3 / 2)_{n}}
$$

As a result, eq. (7-3) reduces to 


$$
\frac{R(t)}{\omega_{p}^{\prime}}=-\frac{1}{2}\left(\frac{\bar{\omega}_{p} t}{2}\right) \sum_{n=0}^{\infty} \frac{1}{(3) n^{n !}}\left[-\left(\frac{{ }_{p} t}{2}\right)^{2}\right]^{n}
$$

$$
=-\frac{1}{2}\left(\frac{{ }_{p}{ }^{t}}{2}\right)_{0} F_{1}\left[3 ;\left(\frac{-\bar{\omega}_{p} t}{2}\right)^{2}\right] \text {. }
$$

By means of identity (6-18) the hypergeometric function appearing in expression (7-5) can be replaced by a second order Bessel function, with the result that for the lossless medium

$$
\frac{R(t)}{\bar{\omega}_{p}}=\frac{-2}{\left(\bar{\omega}_{p} t\right)} J_{2}\left(\bar{\omega}_{p} t\right) .
$$

This particular impulse response function, derived by ather means, has been used to interpret transient returns from ionospheric sounding experiments [8].

The transient propagators for the Drude metal and plasma are obtained from eqs. (7-3) and (7-6) by replacing $t$ with the retarded time function [t] defined in eq. (5-7), as

$$
[t]=t+\left(x \cos \theta_{i}-2 \sin \theta_{i}\right) / c .
$$

A normalized graph of the transient propagator versus retarded time appears in figure 4 for the Drude metal ( $\left.\nu=0.1258 \bar{\omega}_{p}\right)$. The 


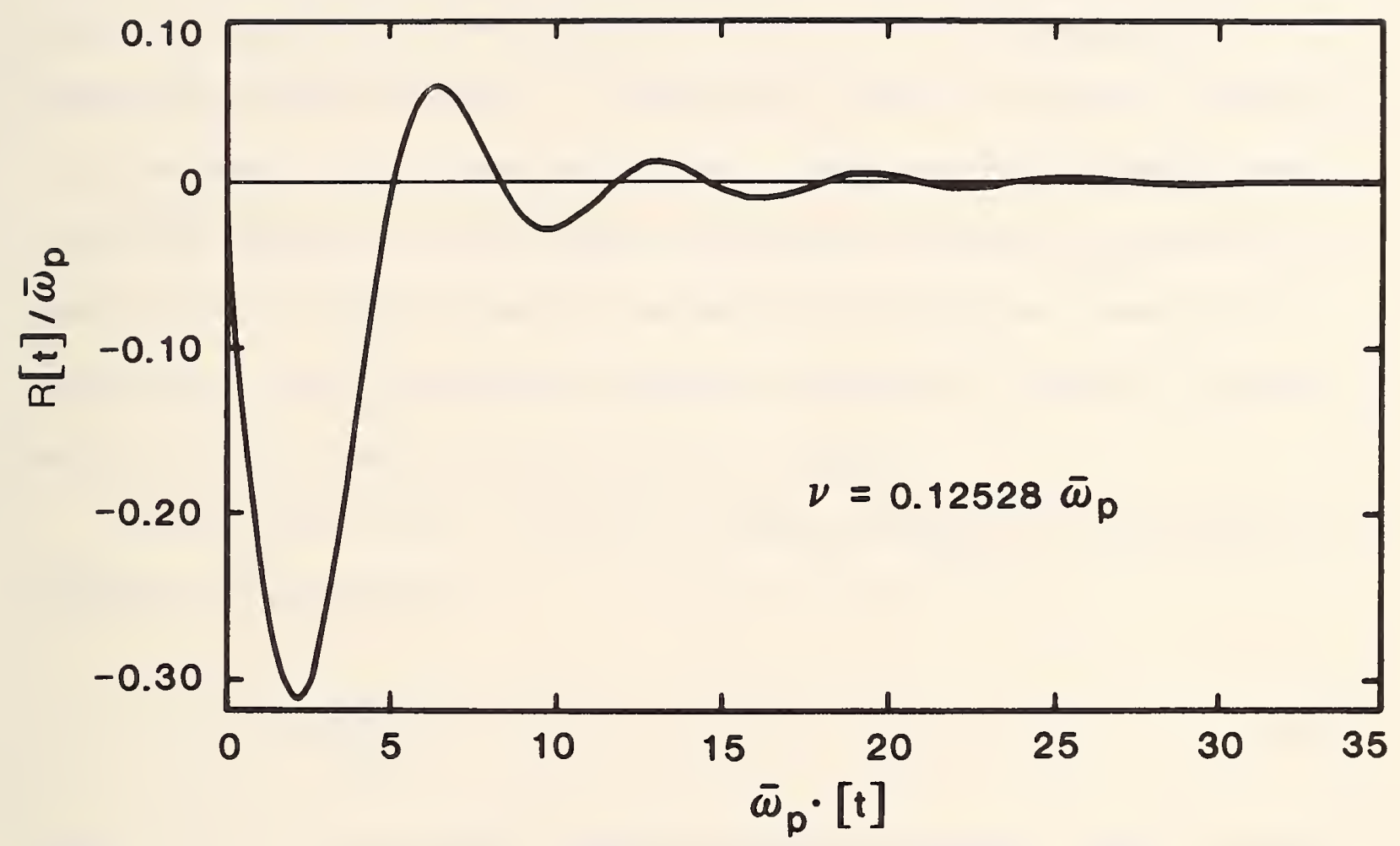

Figure 4. The transient propagator for treating reflections from a Drude metal half-space. The considered value of $v / 6$ corresponds to the underdamped Lorenta dielectric propggator shown in figure $\Xi$ (a) with $\omega_{0}=0$. 
transient propagator for the lossless plasma $(\nu=0)$ has a very similar appearance except that the oscillations are slightly larger and less damped in this case. 
8. THE TRANSIENT PROPAGATOR FOR TREATING REFLECTIONS FROM A DEBYE POLAR DIELECTRIC

The Debye polar dielectric material, typically an organic gas or 1 iquid, is modeled as an ensemble of permanent dipolar molecular elements attributed with rotational freedom. These elements tend to rotate into alignment with an applied field, against opposing thermal forces which tend to randomize the molecular orientations. In comparison with a Lorentz dielectric, a Drude metal, or a neutral plasma, relaxation processes are generally slower in the Debye dielectric because the molecular mass of the rotator greatly exceeds the electron mass of the oscillator and because the thermal restoring forces are generally weaker than those of electrostatic origin.

According to eq. (2-43) the Debye dielectric susceptability function is given by

$$
x(j \omega)=\frac{x(0)}{1+j \omega \tau^{\prime}}
$$

where $x(0)$ is the static susceptibility constant given by eq. (244) and $T^{\prime}$ is related by means of eq. (2-45) to the mean time interval between collisions. For economy of notation the prime appearing on $T$ will hereafter be suppressed; the appearance of $T$ will signify the value of $\tau^{\prime}$ as defined by eq. (2-45).

In order to construct the transient propagator F[t] it is first necessary to replace $j \omega$ by 5 in $(8-1)$ :

$$
x(s)=\frac{X(0) / T}{(5+1 / T)}
$$

and then to define 


$$
\bar{X}(s)=\frac{1}{\bar{\tau}(s+1 / \tau)}
$$

where

$$
\bar{\tau}=\tau \cos ^{2} \theta_{i} / X(0)
$$

Clearly the character of the pole at $s=-1 / \tau$ is not influenced by the magnitude of $\theta_{i}$ or $x(0)$.

In terms of definition (8-3) the transient reflection coefficient determined by eq. (5-4) for susceptibility function $(8-3)$ is

$$
R(s)=\frac{1}{4} \sum_{n=0}^{\infty} \frac{(3 / 2) n}{(3) n}\left[\frac{-1}{\bar{\tau}(s+1 / \tau)}\right]^{n+1} .
$$

Inverting this expression, term by term, by means of the Laplace transform pair $(A+S 29.3 .11):$

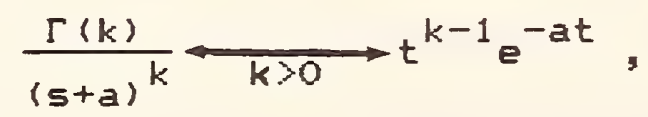

immediately leads to the impulse response function:

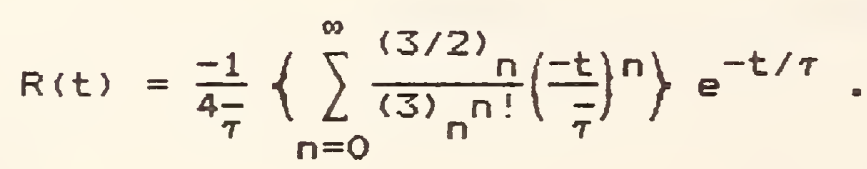

The series contained within the curly brackets is simply the 
confluent hypergeometric function and so

$$
R(t)=\frac{-1}{4 \bar{\tau}}{ }_{1} F_{1}(3 / 2 ; 3 ;-t / \bar{\tau}) e^{-t / \tau}
$$

The confluent hypergeometric function ${ }_{1} F_{1}(a ; b ; z)$ appearing in eq. (8-8) is identical to Kummer's function $M(a, b, z)$. In the notation of Abramowitz and Stegun (A+S 13.1.2 and below 12.1.10):

$$
{ }_{1} F_{1}(3 / 2 ; 3 ;-t / \bar{T}) \equiv M(3 / 2,3,-t / \bar{\tau}) .
$$

Furthermore, it is extremely useful to note that the Kummer function appearing in (8-9) satisfies, with $\nu=1$ and $z=-t / 2 \bar{\tau}$ the identity $(A+513.6 .3)$ :

$$
M(\nu+1 / 2,2 v+1,2 z)=\Gamma(1+\nu)(2 / z){ }^{\nu}{ }_{\nu}(z) e^{z} .
$$

Thus

$$
M(3 / 2,3,-t / \bar{\tau})=-4 \frac{\tau}{t} I_{1}\left(\frac{-t}{2 \bar{\tau}}\right) e^{-t / 2 \bar{\tau}},
$$

where $I_{1}(-t / 2 \bar{\tau})$ is the modified Bessel function. Substitution of eqs. (8-9) and $(8-11)$ into eq. (8-8) now yields

$$
R(t)=+\frac{1}{t} I_{1}\left(\frac{-t}{2 \tau}\right) \exp \left[-\left(\frac{1}{\tau}+\frac{1}{2 \bar{\tau}}\right) t\right] \text {. }
$$

Since $I_{1}(-z)=-I_{1}(z)$, the impul se response function for the Debye dielectric assumes as its final form: 


$$
\tau R(t)=-\frac{\tau}{t} I_{1}\left(\frac{t}{2 \tau}\right) \exp \left[-\left(\frac{1}{\tau}+\frac{1}{2 \bar{\tau}}\right) t\right] .
$$

The transient propagator is obtained by replacing $t$ in this expression by relation (5-7) for [t]. The behavior of R[t] is graphed in figure 5 for several values of $\tau / \bar{T}$. 


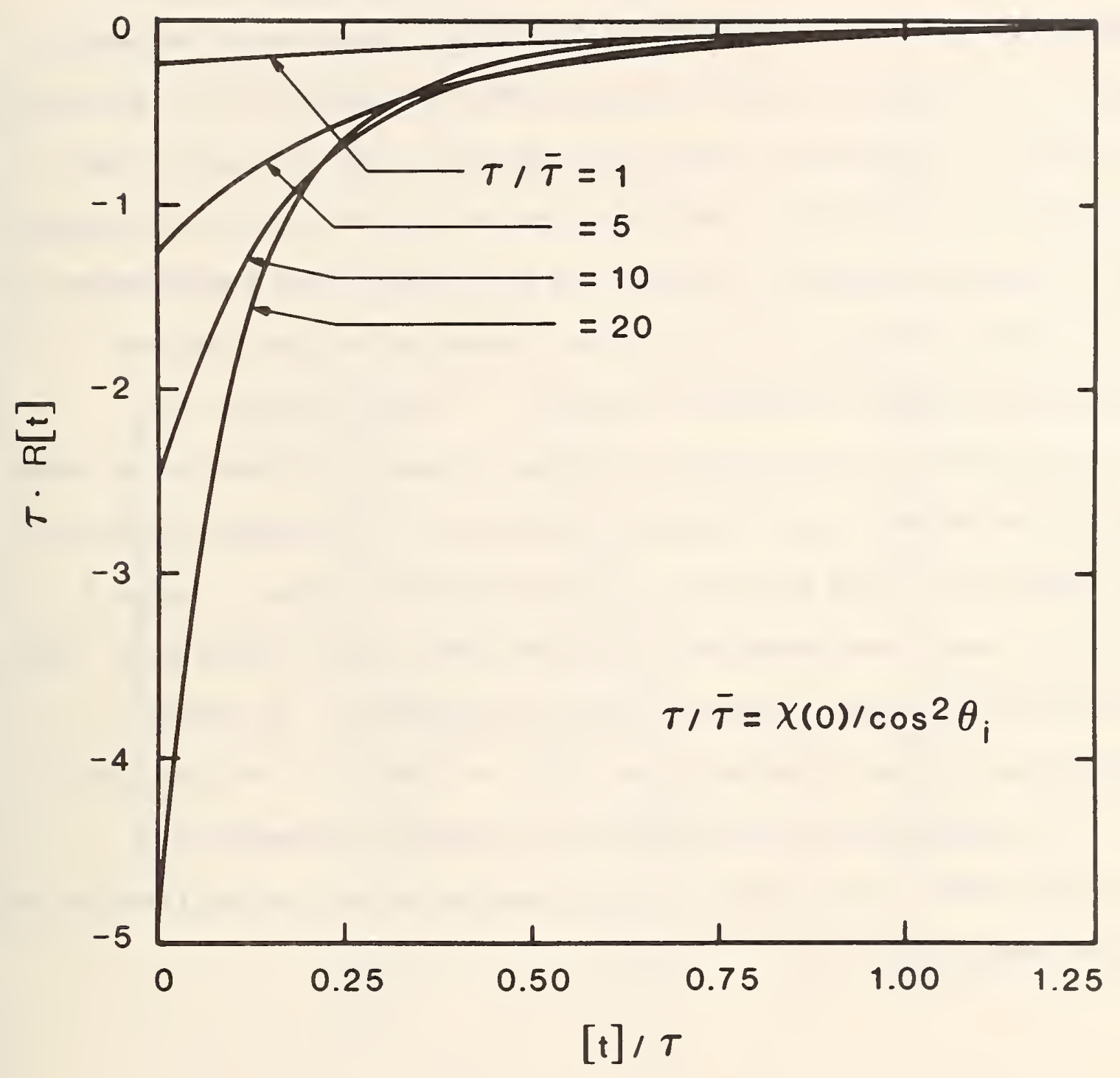

Figure 5. The transient propagator for treating reflections from a Debye dielectric half-space. Four cases are shown, each parametrized by a different value of the static susceptability function $x(0)$ divided by the squared cosine function of the incidence angle $\theta_{i}$. 


\section{SUMMARY AND CONCLUSION}

The transient propagator (or Green"s function) dealing with the reflection of an incident, impulsive, plane wave is the fundamental solution to the time-dependent plane wave reflection problem. By means of this function the reflection of an incident plane wave of arbitarily specified waveform can be calculated via a convolution integral. Construction of the transient propagator for a transverse-electric polarized plane wave was implemented by an algorithm devised to facilitate inversion of the Laplace transformed impulse response function. This algorithm was applied to study reflections from four frequency-dispersive model material surfaces: the Lorentz dielectric: the Debye dielectric; the Drude metal; and the cold, collisionless plasma. Table 1 lists the impulsive response functions which were obtained. The transient propagators, determined by replacing $t$ in these expressions by the retarded time [t], are shown in figures 3,4 , and 5. Corresponding expressions for transverse-magnetic polarized fields, or other material surfaces may be derived using similar techniques. 

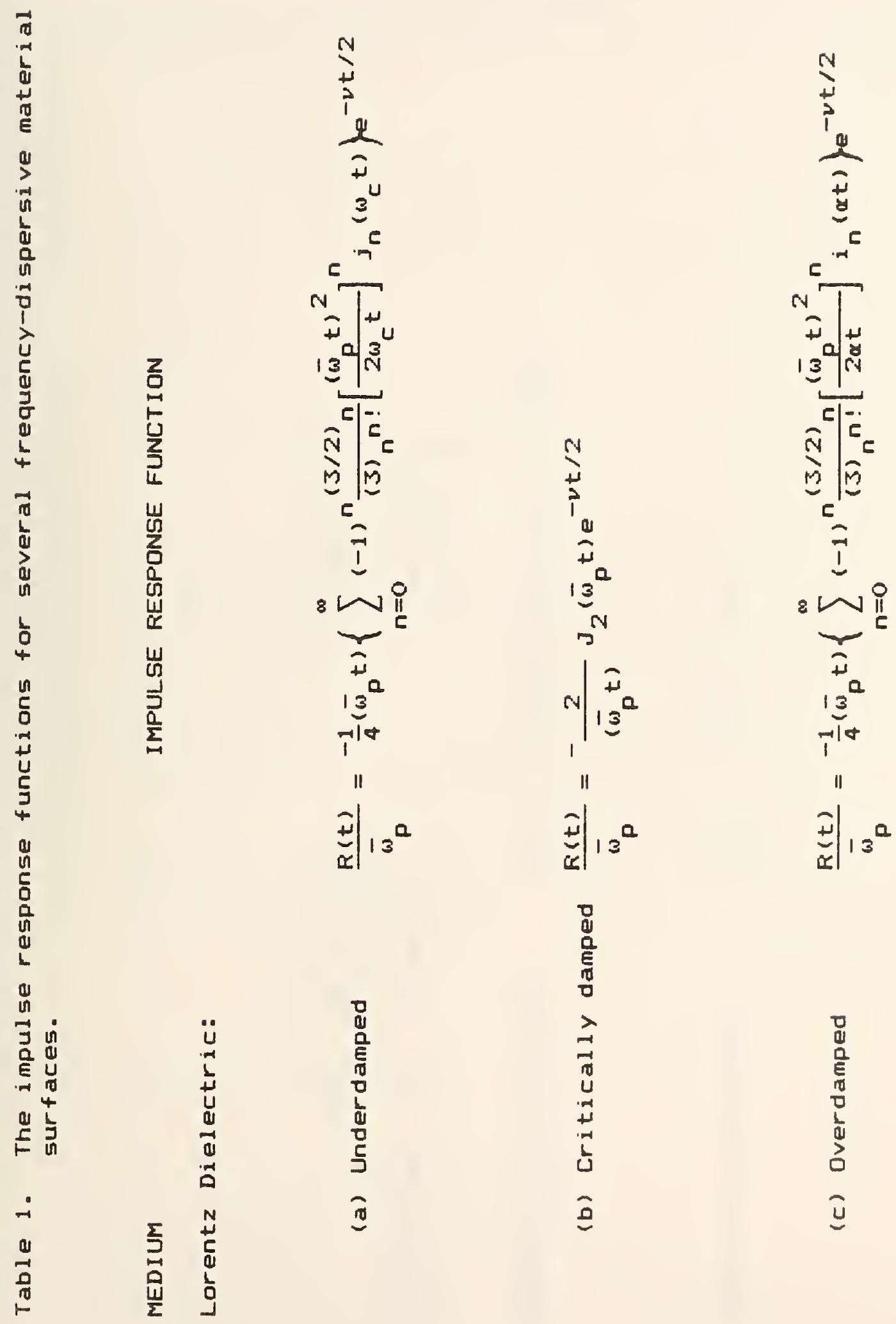


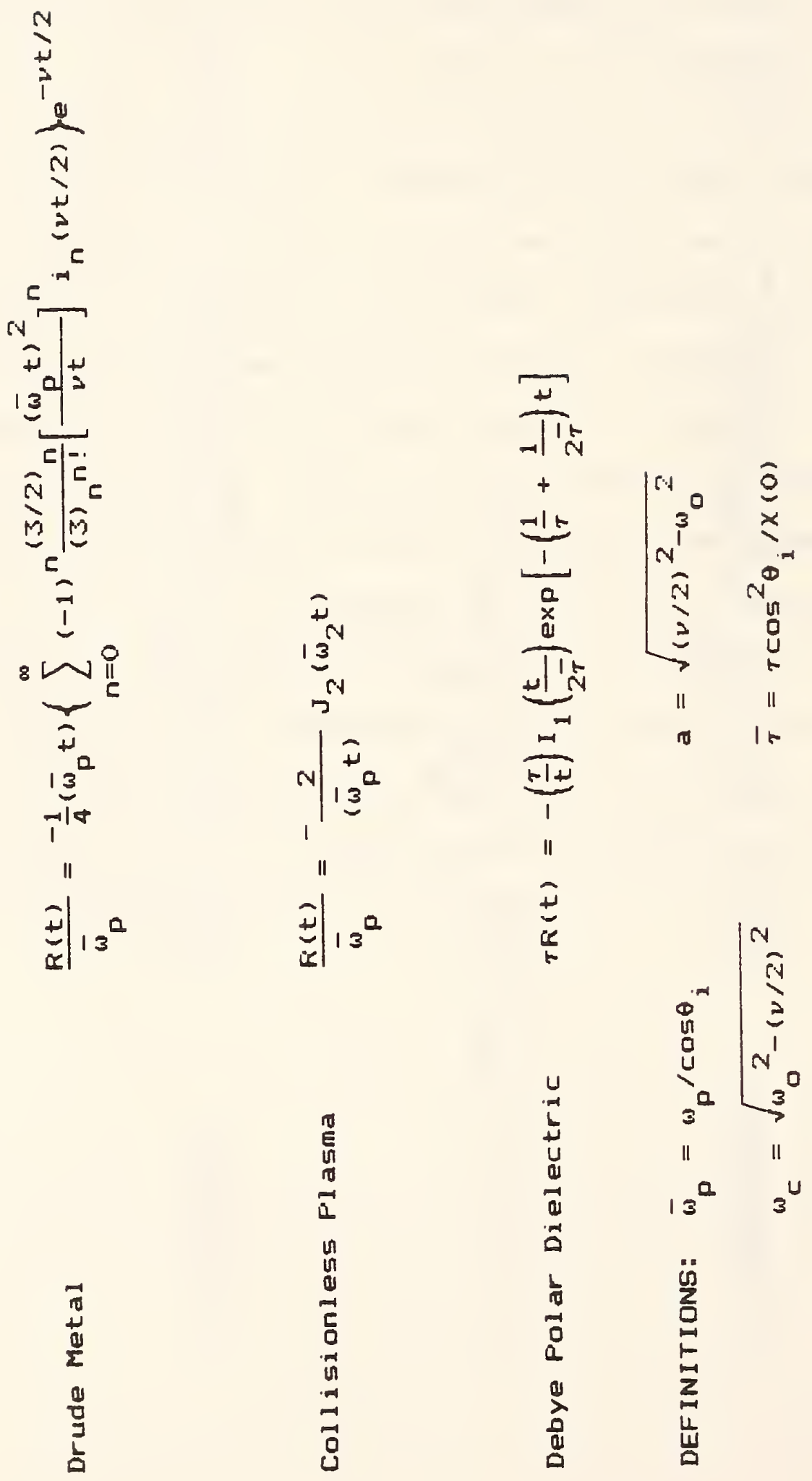


10. ACKNOWLGEMENT

The author wishes to thank J. F. Marchiando for performing the numerical computations leading to figures 3, 4, and 5 and for providing many helpful suggestions throughout this project. 
11. REFERENCES

1. Fork, R. L.; Shank, C. V.; Yen, R.; Hirlimann, C. A. Femtosecond optical pulses. IEEE Journal of Quantum Electronics QE-1q⒁: 500-505; 1983.

2. Brillouin, L. Wave propagation and group velocity. New York: Academic Press; 1960. 154 p.

3. Wait, J. R.; Froese, C. Reflection of a transient electromagnetic wave at a conducting surface. Journal of Geophysical Research $6 \underline{6}(1): 97-103: 1955$.

4. Dudley, D. G.; Papazoglou, T. M.; White, R. C. On the interaction of a transient electromagnetic plane wave and a lossy half-space. Journal of Applied Physics 4ㅗ( 3 ): $1171-$ $1175 ; 1974$.

5. Chen, S. N. C. The transient phenomenon in an isotropic plasma without collision loss. Proceedings of the IEEE $\underline{5}(7): 1045-1046 ; 1963$.

6. Knop, C. M. Comments on "The transient phenomenon in an isotropic plasma wi thout collision loss." Proceedings of the IEEE $5 \underline{2}(1)$ : $99 ; 1964$.

7. Knop, C. M. Further comments on "The transient phenomenon in an isotropic plasma without collision loss." Proceedings of the IEEE $5 \underline{3}(7): 751-752 ; 1965$.

8. Wait, J. R. Oblique reflection of a plane impulsive electromagnetic wave from a plasma half-space. Physics of Fluids 12 (7): $1521-1522 ; 1969$.

9. Price, G. H. Reflection at oblique incidence of an impulsive transverse magnetic plane wave from a cold, lossy. semi-infinite plasma. Radio Science $\underline{8}(12): 1165-1168 ; 1973$. 
10. Debye, P. Zur theorie der anomalen Dispersion in Gebiete der langwelligen elektrischen Strahlung. Verhandlungen der Deutsche Physikalischen Gesellschaft 15(16): 777-793: 1913.

11. Lorentz, H. A. The theory of electrons and their applications to the phenomena of 1 ight and radiant heat.

Znd ed. New York: G. E. Stechert and Co.; 1916. Chapt. IV, 132-167; Lecture note 57, 309-311.

12. Drude, P. Zur Ionen-Theorie der Metalle. Physikalische Zeitschrift 1 (14): 161-165; 1900 .

13. Kramer5, H. A.; Heisenberg, W. Ueber die Streuung von Strahlung durch Atome. Zeitschrift fuer Fhysik 31 (9): 681$708 ; 1925$.

14. Lorentz, H. A. Ueber die Beziehung zwischen der Fortpflanzungsgeschwindigkeit des Lichtes und der Koerpersdichte. Weidemann, Annalen der Physik q(4): 641-665; 1880.

15. Lorenz, L. Ueber die Refractionsconstante. Weidemann, Annalen der Physik 11 (9): 70-103; 1880.

16. Born, M.; Walf, E. Principles of Dptics, bth ed. New York: Pergamon Pres5; 1980. 36-51. 



1. PUBLICATION OR
REPORT NO.
NBS / TN-1202

2. Performing Organ. Report Nod 3. Publication Date

BIBLIOGRAPHIC DATA

SHEET (See instructions)

NBS / TN-1202

March 1985

4. TITLE AND SUBTITLE

Transient Analysis of Electromagnetic Reflection From Dispersive Materials

5. $A \cup T H O R(S)$

A. George Lieberman

6. PERFORMING ORGANIZATION (If joint or other thon NBS, see instructions)

7. ContracUGrant No.

National Bureau of Standards

Department of Commerce

Gaithersburg, MD 20899

8. Type of Report \& Period Covered

Final

9. SPONSORING ORGANIZATION NAIEE AND COMPLETE ADDRESS (Strech, Cll, StOte, ZIP)

Same as Item 6

10. SUPPLEMENTARY NOTES

[D Document describes a computer program; SF-185, FIPS Software Summary, is attached.

11. ABSTRACT (A 200-word or less factual summary of most significant information. If document includes a significant bibliography or literature survey, mention it here)

Theoretical expressions are presented describing the transient and steady-state temporal evolution of an impulsive, TE-polarized, plane wave reflected into vacuum from any of a variety of frequency-dispersive material surfaces. Polar dielectrics, non-polar dielectrics, metals and plasmas, are treated using, respectively, the Debye, Lorentz, and Drude material models to investigate the effects of dispersion upon dimensional measurements performed with optical pulses of extremely short duration. The more general problem, concerning the reflection of an optical pulse of any specified waveform, is resolved by convoluting the incident wave at the material surface with the previously determined reflection of an impulsive wave.

12. KEY WORDS (Six to twelve entries: alphabetical order; capitalize only proper names; and separate key words by semicolons) Debye dielectric; dispersion; Drude metal; electromagnetic fields; Green's function; impulse response; Lorentz dielectric; material models; optical pulses; plasma; reflected waves; transient fields; waveform analysis

13. AVAILABILITY

[X] Unlimited

For Official Distribution. Do Not Release to NTIS

[X] Order From Superintendent of Documents, U.S. Government Printing Office, Washington, D.C. 20402.

14. NO. OF

PRINTED PAGES

Order From National Technical Information Service (NTIS), Springfield, VA, 2216I 



\section{Technical Publications}

\section{Periodical}

Journal of Research-The Journal of Research of the National Bureau of Standards reports NBS research and development in those disciplines of the physical and engineering sciences in which the Bureau is active. These include physics, chemistry, engineering, mathematics, and computer sciences. Papers cover a broad range of subjects, with major emphasis on measurement methodology and the basic technology underlying standardization. Also included from time to time are survey articles on topics closely related to the Bureau's technical and scientific programs. As a special service to subscribers each issue contains complete citations to all recent Bureau publications in both NBS and non-NBS media. Issued six times a year.

\section{Nonperiodicals}

Monographs-Major contributions to the technical literature on various subjects related to the Bureau's scientific and technical activities.

Handbooks-Recommended codes of engineering and industrial practice (including safety codes) developed in cooperation with interested industries, professional organizations, and regulatory bodies.

Special Publications-Include proceedings of conferences sponsored by NBS, NBS annual reports, and other special publications appropriate to this grouping such as wall charts, pocket cards, and bibliographies.

Applied Mathematics Series-Mathematical tables, manuals, and studies of special interest to physicists, engineers, chemists, biologists, mathematicians, computer programmers, and others engaged in scientific and technical work.

National Standard Reference Data Series-Provides quantitative data on the physical and chemical properties of materials, compiled from the world's literature and critically evaluated. Developed under a worldwide program coordinated by NBS under the authority of the National Standard Data Act (Public Law 90-396).

NOTE: The Journal of Physical and Chemical Reference Data (JPCRD) is published quarterly for NBS by the American Chemical Society (ACS) and the American Institute of Physics (AIP). Subscriptions, reprints, and supplements are available from ACS, 1155 Sixteenth St., NW, Washington, DC 20056.

Building Science Series-Disseminates technical information developed at the Bureau on building materials, components, systems, and whole structures. The series presents research results, test methods, and performance criteria related to the structural and environmental functions and the durability and safety characteristics of building elements and systems.

Technical Notes-Studies or reports which are complete in themselves but restrictive in their treatment of a subject. Analogous to monographs but not so comprehensive in scope or definitive in treatment of the subject area. Often serve as a vehicle for final reports of work performed at NBS under the sponsorship of other government agencies.

Voluntary Product Standards-Developed under procedures published by the Department of Commerce in Part 10, Title 15, of the Code of Federal Regulations. The standards establish nationally recognized requirements for products, and provide all concerned interests with a basis for common understanding of the characteristics of the products. NBS administers this program as a supplement to the activities of the private sector standardizing organizations.

Consumer Information Series-Practical information, based on NBS research and experience, covering areas of interest to the consumer. Easily understandable language and illustrations provide useful background knowledge for shopping in today's technological marketplace.

Order the above NBS publications from: Superintendent of Documents, Government Printing Office, Washington, DC 20402.

Order the following NBS publications-FIPS and NBSIR's-from the National Technical Information Service, Springfield, VA 22161 .

Federal Information Processing Standards Publications (FIPS PUB)-Publications in this series collectively constitute the Federal Information Processing Standards Register. The Register serves as the official source of information in the Federal Government regarding standards issued by NBS pursuant to the Federal Property and Administrative Services Act of 1949 as amended, Public Law 89-306 (79 Stat. 1127), and as inplemented by Executive Order 11717 (38 FR 12315, dated May 11, 1973) and Part 6 of Title 15 CFR (Code of Federal Regulations).

NBS Interagency Reports (NBSIR)-A special series of interim or final reports on work performed by NBS for outside sponsors (both government and non-government). In general, initial distribution is handled by the sponsor; public distribution is by the National Technical Information Service, Springfield, VA 22161, in paper
copy or microfiche form. 
U.S. Department of Commerce National Bureau of Standards

Gaithersburg, MD 20899

Official Business

Penalty for Private Use $\$ 300$ 